\title{
Variation in quartzite exploitation during the Upper Palaeolithic of Southwest Iberian Peninsula
}

\author{
Variación en la explotación de la cuarcita durante el Paleolítico Superior en el Suroeste \\ de la Península Ibérica
}

Telmo Pereira (*)

João Cascalheira $(*)$

João Marreiros (*)

Francisco Almeida (**)

Nuno Bicho(**)

\begin{abstract}
The Upper Paleolithic of SW Iberia is marked by the presence of chopper and flake assemblages in quartzite. Detailed characterization at regional and chronological levels of these assemblages is of the utmost importance because, in the most Paleolithic recent phases, they can be found without type-fossils associated or in non-datable deposits. In this study, we used 24 quartzite assemblages from SW Iberia, to test the diagnostic character of this raw material through attribute analysis and refitting. Results indicate that Gravettian, Solutrean and Magdalenian can be distinguished on their quartzite assemblages, enabling, by itself, the differentiation of the Upper Paleolithic key-sequence. They also indicate that Gravettian and Magdalenian assemblages are technologically closer to each other than to Solutrean, a pattern possibly related with the adaptation to the Last Glacial Maximum.
\end{abstract}

\section{RESUMEN}

El Paleolitico Superior del Suroeste de la Península Ibérica está marcado por la presencia de conjuntos de cantos tallados y lascas en cuarcita. La caracterización detallada a nivel regional y cronológico de estos conjun-

(*) Núcleo de Arqueologia e Paleoecologia (NAP). Faculdade de Ciências Humanas e Sociais, Universidade do Algarve (Ulag) - Campus Gambelas. 8005-139 Faro. Portugal. La página web de Ualg está en actualización. El NAP podrá ser identificado en http://www.ualg.pt/index.php?option=com_content\& task $=$ view\&id $=25450 \&$ Itemid $=2718 \&$ lang $=p t$

E-mail: telmojrpereira@gmail.com

(**) Dept. of Planning and Community Development. Aboriginal Affairs Victoria. GPO 2392 Melbourne VIC 3001 Australia.

Received: 9-XII-2011; accepted: 13-II-2012. tos es de la mayor importancia, ya que, en las fases más recientes del Paleolítico, se pueden encontrar sin asociación a fósiles-guía o depósitos datables. En este estudio, hemos utilizado 36 conjuntos de cuarcita de esa región para poner a prueba el carácter diagnóstico de esta materia prima a través de análisis de tributos y remontajes. Los resultados indican que Gravetiense, Solutrense y Magdaleniense se pueden distinguir en sus conjuntos de cuarcita, lo que permite, por sí mismo, la diferenciación de la secuencia clave del Paleolítico Superior. También indican que los conjuntos Gravetiense y Magdaleniense están tecnológicamente más próximos entre sí que respecto al Solutrense, un patrón posiblemente relacionado con la adaptación al Último Máximo Glaciar.

Key words: Chronological variation; Coarse raw materials; Lithic technology; Southeast of the Iberian Peninsula Iberia; Upper Paleolithic.

Palabras clave: Variación cronológica; Materias-primas granulosas; Tecnología lítica; Sureste de la Península Ibérica; Paleolítico Superior.

\section{INTRODUCTION}

Chopper and flake assemblages on non-flint raw materials are worldwide known and omnipresent through all diachrony of hominid tool production. In the case of the Portuguese Upper Paleolithic and Epipaleolithic, their crude aspect and often non prepared reduction is a clear deceiver of the intrinsic complexity of their production. This was shown, for instance, by refitting at Barca do Xerêz (Araújo and Almeida 2007), Lagar Velho (Zilhão and Almeida 2002), Gato 
Preto, Cabeço de Porto Marinho or Anecrial Cave (Almeida 2000). Therefore, a detailed project dedicated to their comprehensive characterization through the Upper Paleolithic was of most importance in order to recognize regional and chronological variations. They represent one of the most important distinctiveness of the Western Iberian Paleolithic, a phenomenon noted right from the beginning of archaeological investigation in Portugal (Ribeiro 1871). The Portuguese Upper Paleolithic is represented by several sub-periods, distinguished by the presence of very different leptolithic technologies and diagnostic tools (Zilhão 1997; Bicho 2000), with flint dominating the quarry sites but with considerable important quantities of quartz and quartzite in both residential and logistic campsite. Elucidation of the Upper Paleolithic sub-divisions was the result of 150 years of intensive research in several Portuguese regions. Typological and technological analyses revealed that both blank production and retouchedtool types closely followed the traditional Western European patterns and tool-kits. Blades were mostly produced on flint through prismatic cores which preparation included descortification, configuration and the creation of a crest from where the blanks begun to be extracted; reshape was common. Bladelets are usually in flint and quartz. This type of blank was extracted not only through prepared prismatic cores, but also through thick endscrapers and carinated burins. In the first case, the result tended to be twisted blanks, while in the second the result was the creation of thick blanks, also known as burin spalls. These studies brought about the local recognition of several sub-stages of the Early Gravettian, Middle, Late and Terminal Gravettian; Proto and Upper Solutrean, Early, Middle, Final and Terminal Magdalenian. The combination of stratigraphy, artifact seriation with absolute dating allowed the clarification of their temporal organization, resulting in the creation of a fairly accurate Western Iberian Upper Paleolithic key-sequence (Heleno 1956, Marks et al. 1994; Bicho 1996, 2000, 2001, 2004; Zilhão 1997; Gameiro and Almeida 2001; Zilhão and Almeida 2002; Almeida et al. 2002; Almeida et al. 2004: Almeida et al. 2008; Aubry 2009; Cascalheira 2010).

During the Upper Paleolithic, quartzite components are mostly regarded as composed of choppers and flakes, presenting cortex and usually lacking any kind of preparation. Elongated products, diagnostic tool-types (which are those in which most of the traditional key-sequence is based), and weaponry are scarce or nonexistent, and, traditionally seen as without unique technological strategies or diachronic differences. This is completely different from the scenario prior to the emergence of the Upper Paleolithic, when non-flint raw materials are present in cores, blanks, retouched and shaped diagnostic tools. Traditionally, this differentiated use of quartzite in the Upper Paleolithic had two different interpretations. Some considered it as reflecting an opportunistic use of local raw material in order to substitute or preserve flint (Zilhão 1997), while others (Bicho 2000) suggested that it might have been used to perform some specific domestic functions, although, not specifying what. Both perspectives seem to agree on the fact that the reason behind the difference between flint/quartz and quartzite assemblages might have been the availability or/and the low quality of the latter for the production of blades and bladelets. This would be explained by the relatively low frequency of these quartzite blanks in the assemblages of such period (Bicho 1996, 2000; Zilhão 1996, 1997). However, very thin, long and sometimes exquisite products such as handaxes in the Micoquian or Levallois blanks, including elongate flakes and blades in the Mousterian are frequent. Independently of the reasons, despite the presence of a large number of sites dominated by chopper and flake industries that lack the traditional type-fossils (Breuil and Zbyszewski 1942, 1945; Zbyszewski 1966; Raposo and Silva 1984; Raposo 1986; Meireles 1992), the impasse on decoding the post-Middle Paleolithic quartzite components resulted in the decrease of the study of such assemblages.

The recent discovery of new sites, some of which are close to important flint outcrops, in which quartzite and greywacke are abundant and often dominating the assemblages (Almeida et al. 2002; Almeida et al. 2008; Araújo and Almeida 2007; Aubry 2009), has re-opened discussion on the interpretation of quartzite exploitation as merely opportunistic. At the same time, study of these new data revealed that the reduction sequences were quite complex. 


\section{METHODS AND MATERIALS}

To test the importance and variability of coarse raw materials in the tool-kits from Anatomically Modern Human communities, we used SW Iberia as a study case, by applying a method that combines two lithic technology approaches (attribute based analysis and refitting), statistical enquiry and refitting. The objectives were:

1. To infer if the importance of quartzite as raw material for the anatomically modern human groups in this region.

2. If its exploitation and use changed between the beginning of the Gravettian and the end of the Magdalenian.

3. To construct, if possible, a consistent model for the quartzite exploitation during the Upper Paleolithic, in SW Iberia.

The region approached was Central Portugal. We selected 10 sites, corresponding to 24 archaeological contexts (Fig. 1, Tab. 1).

Dated to the Gravettian are Caldeirão Cave (layers Jb, Ja, I, H), Terra do Manuel, Fonte Santa and Alecrim Rockshelter. The Solutrean assemblages also came from Caldeirão Cave $(\mathrm{Fa}, \mathrm{Fb}, \mathrm{Fc}$, H) and Casal do Cepo. Finally, the Magdalenian ones came from Caldeirão Cave (layers Ea, Ebtop, Eb-bottom), Picareiro Cave (layers E, F, G, I, $\mathrm{J}, \mathrm{K}, \mathrm{L}$ ), Bocas (layer Bottom) and Bairrada. Available absolute dates are presented in table 2. De-

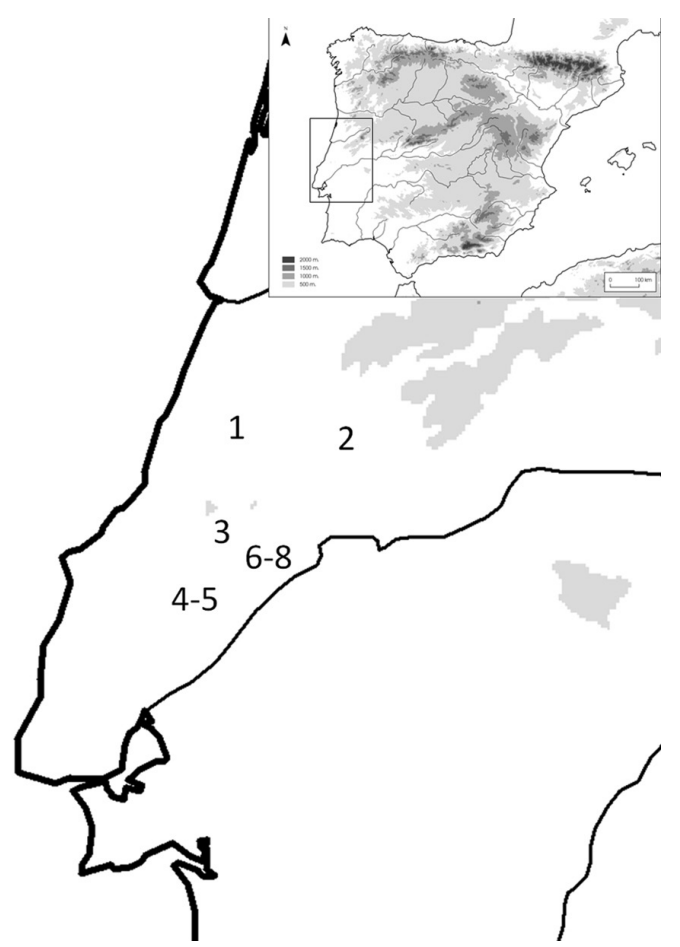

Fig. 1. Map of Iberia Peninsula with the identification of the sites studied in Estremadura: 1. Alecrim Rockshelter (Leiria); 2. Caldeirão Cave (Tomar); 3. Picareiro Cave (Alcanede); 4. Bocas Rockshelter (Rio Maior); 5. Terra do Manuel (Rio Maior); 6. Bairrada (Torres Novas); 7. Casal do Cepo (Torres Novas); 8. Fonte Santa (Torres Novas).

\begin{tabular}{|c|c|c|c|c|c|c|c|c|c|c|c|c|c|c|c|c|c|c|c|c|c|c|c|c|}
\hline Complex & & & & Grave & ian & & & & Solu & rean & & & & & & & Tagdal & lenian & & & & & & \\
\hline Site & 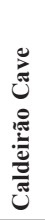 & 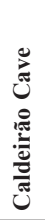 & 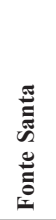 & 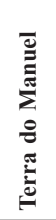 & 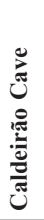 & 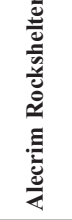 & 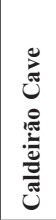 & 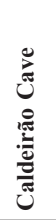 & 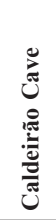 & 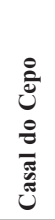 & 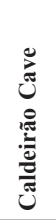 & 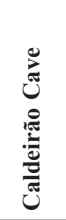 & 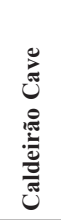 & 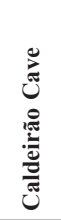 & 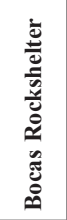 & 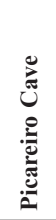 & 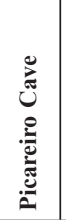 & 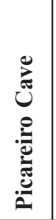 & 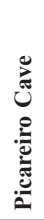 & 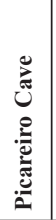 & 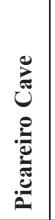 & 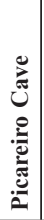 & 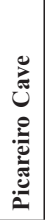 & 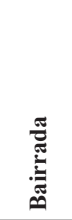 \\
\hline Layer & $\mathbf{J b}$ & $\mathbf{J a}$ & & & I & & H & Fe & Fb & & $\mathbf{F a}$ & $\begin{array}{c}\text { Eb- } \\
\text { base }\end{array}$ & $\begin{array}{l}\text { Eb- } \\
\text { top }\end{array}$ & Ea & $\begin{array}{l}\text { Bot- } \\
\text { tom }\end{array}$ & E & F & G & H & I & $\mathbf{J}$ & $\mathbf{K}$ & $\mathbf{L}$ & \\
\hline Pebbles & 1 & & 2 & 4 & & 100 & & 1 & 2 & 4 & 2 & 1 & 2 & 1 & 2 & 3 & 2 & & & & & & & 7 \\
\hline Cores & & 2 & 2 & 33 & 1 & 30 & 1 & 3 & 4 & 5 & 24 & 37 & 17 & 29 & 16 & 9 & 5 & 1 & & & 2 & & & 19 \\
\hline Flakes & 13 & 23 & 631 & 716 & 22 & 292 & 107 & 68 & 107 & 450 & 337 & 121 & 108 & 447 & 35 & 57 & 60 & 31 & & 8 & 5 & & & 892 \\
\hline Blades & & 2 & 24 & 2 & 1 & 6 & & 4 & 12 & 17 & 14 & 7 & & 12 & & 3 & & 2 & & 18 & & & & \\
\hline Bladelet & & 4 & 10 & 5 & 1 & 24 & & 1 & 10 & 1 & 16 & 2 & & 15 & & 2 & & & 1 & 2 & 1 & & & 68 \\
\hline Maintenance & & 3 & & & 3 & 5 & & & 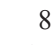 & 41 & 9 & 28 & & & & & & & & & & & & \\
\hline Fragments & 2 & & 68 & 46 & 1 & 120 & & 16 & 15 & 28 & 34 & 35 & 14 & 36 & & 3 & 2 & & & 31 & & & & \\
\hline Chips & & 9 & 33 & 113 & 4 & 369 & 1 & 9 & 24 & 4 & 33 & 28 & 6 & 47 & & 366 & 69 & 38 & 3 & 20 & 4 & 5 & 2 & 232 \\
\hline Fire cracks & 2 & 1 & 58 & 68 & & 124 & 10 & 20 & 36 & 9 & 47 & 96 & 4 & 47 & & 4 & 3 & & & 1 & & & & \\
\hline Total & 18 & 44 & 854 & 987 & 33 & 1.070 & 119 & 122 & 218 & 569 & 516 & 327 & 151 & 634 & 53 & 447 & 141 & 72 & 4 & 31 & 12 & 5 & 2 & 1.295 \\
\hline
\end{tabular}

Tab. 1. Quartzite inventories of from each studied archaeological context, approximately organized from older (left) to recent (right). 


\begin{tabular}{|c|c|c|c|c|c|}
\hline & Sample & Layer & Material & $\begin{array}{c}\text { Date (BP - non } \\
\text { calibrated) }\end{array}$ & Reference \\
\hline Fonte Santa & $\begin{array}{l}\text { FS A7 } \\
\text { FS A15 } \\
\text { FS A3 }\end{array}$ & $\begin{array}{l}\text { T43 }(-28 \mathrm{~cm}) \\
\text { Q45 }(-30 \mathrm{~cm}) \\
\text { P42 }(-35 \mathrm{~cm})\end{array}$ & $\begin{array}{l}\text { Burned flint } \\
\text { Burned flint } \\
\text { Burned flint }\end{array}$ & $\begin{array}{l}40,400 \pm 4600(\mathrm{r}) \\
39,300 \pm 4700(\mathrm{r}) \\
35,300 \pm 3600(\mathrm{r})\end{array}$ & $\begin{array}{l}\text { Zilhão } 1997 \\
\text { Zilhão } 1997 \\
\text { Zilhão } 1997\end{array}$ \\
\hline Terra do Manuel & $\begin{array}{c}\text { ETH- } 6038 \\
\text { TL } \\
\text { TL } \\
\text { TL }\end{array}$ & $\begin{array}{l}2 s \\
2 \\
2 \\
2\end{array}$ & $\begin{array}{l}\text { Carvão } \\
\text { Burned flint } \\
\text { Burned flint } \\
\text { Burned flint }\end{array}$ & $\begin{array}{c}21,700 \pm 210 \\
7,300 \pm 700(\mathrm{r}) \\
15,700 \pm 170(\mathrm{r}) \\
16,400 \pm 1800(\mathrm{r})\end{array}$ & $\begin{array}{l}\text { Zilhão } 1997 \\
\text { Zilhão } 1997 \\
\text { Zilhão } 1997 \\
\text { Zilhão } 1997\end{array}$ \\
\hline Alecrim Rockshelter & \begin{tabular}{|c|} 
Wk 23517 \\
Wk 23515 \\
Wk 23514 \\
Beta: 203513 \\
\end{tabular} & $\begin{array}{l}6 \\
6 \\
6 \\
6 \\
\end{array}$ & $\begin{array}{c}- \\
\text { Quercus pyrenaica } \\
\text { Bone } \\
\text { Bone } \\
\end{array}$ & $\begin{array}{c}\text { Failled } \\
4,632 \pm 32(\mathrm{r}) \\
21,794 \pm 170 \\
20,510 \pm 150 \\
\end{array}$ & $\begin{array}{l}\text { Pereira } 2010 \\
\text { Pereira } 2010 \\
\text { Pereira } 2010 \\
\text { Pereira } 2010 \\
\end{array}$ \\
\hline Caldeirão Cave & $\begin{array}{l}\text { ICEN-72 } \\
\text { ICEN-70 } \\
\text { ICEN-69 } \\
\text { ICEN-295 } \\
\text { OxA-1938 } \\
\text { OxA-2510 } \\
\text { OxA-1939 } \\
\text { OxA-2511 } \\
\text { OxA-1940 } \\
\text { OxA-5542 }\end{array}$ & $\begin{array}{l}\text { Eb-top } \\
\text { Eb-base } \\
\text { Fa pit } \\
\text { Fa top } \\
\text { Fa top } \\
\text { Fc } \\
\text { H } \\
\text { H } \\
\text { I } \\
\text { Jb }\end{array}$ & $\begin{array}{l}\text { Bone } \\
\text { Bone } \\
\text { Bone } \\
\text { Charcoal } \\
\text { Bone } \\
\text { Bone } \\
\text { Bone } \\
\text { Bone } \\
\text { Bone } \\
\text { Charcoal }\end{array}$ & $\begin{array}{c}10,700 \pm 380 \\
14,450 \pm 890 \\
18,341 \pm 1573 \\
20,400 \pm 207(\mathrm{r}) \\
20,40 \pm 270 \\
18,840 \pm 200 \\
19,900 \pm 260 \\
20,530 \pm 270 \\
22,900 \pm 380 \\
26,020 \pm 320\end{array}$ & $\begin{array}{l}\text { Zilhão } 1997 \\
\text { Zilhão } 1997 \\
\text { Zilhão } 1997 \\
\text { Zilhão } 1997 \\
\text { Zilhão } 1997 \\
\text { Zilhão } 1997 \\
\text { Zilhão } 1997 \\
\text { Zilhão } 1997 \\
\text { Zilhão } 1997 \\
\text { Zilhão } 1997\end{array}$ \\
\hline Picareiro Cave & $\begin{array}{c}\text { Wk-4217 } \\
\text { Wk-5431 } \\
\text { Wk10434 } \\
\text { Wk4218 } \\
\text { Wk-4219 } \\
\text { Wk-6677 } \\
\text { OxA-5527 } \\
\text { Wk-10433 } \\
\text { Wk-6678 }\end{array}$ & $\begin{array}{c}\text { E Upper } \\
\text { E Middle } \\
\text { E Middle } \\
\text { E Lower } \\
\text { F } \\
\text { F } \\
\text { G } \\
\text { J } \\
\text { J }\end{array}$ & $\begin{array}{l}\text { Charcoal } \\
\text { Charcoal } \\
\text { Charcoal } \\
\text { Charcoal } \\
\text { Charcoal } \\
\text { Charcoal } \\
\text { Charcoal } \\
\text { Charcoal } \\
\text { Charcoal }\end{array}$ & $\begin{array}{c}10,070 \pm 80 \\
11,700 \pm 120 \\
12,500 \pm 160(\mathrm{r}) \\
11,550 \pm 120 \\
11,780 \pm 90 \\
12,210 \pm 100 \\
12,320 \pm 90 \\
10,490 \pm 110(\mathrm{r}) \\
11,880 \pm 80(\mathrm{r})\end{array}$ & $\begin{array}{l}\text { Bicho et al. } 2003 \\
\text { Bicho et al. } 2003 \\
\text { Bicho et al. } 2003 \\
\text { Bicho et al. } 2003 \\
\text { Bicho et al. } 2003 \\
\text { Bicho et al. } 2003 \\
\text { Bicho et al. } 2003 \\
\text { Bicho et al. } 2003 \\
\text { Bicho et al. } 2003\end{array}$ \\
\hline Bocas Rockshelter & ICEN-901 & Bottom & Bone & $10,110 \pm 90$ & Bicho 1997 \\
\hline
\end{tabular}

Tab. 2. Absolute dates from the archaeological contexts studied in this paper(1).

tailed description and inventories of each context can be seen elsewhere (Bicho 1997; Zilhão 1997; Quelhas 1999; Bicho et al. 2003; Pereira 2010).

The analyses follow the common lithic technology concepts used in Western Europe (e.g., Inizan et al. 1999). Assemblages were divided as shown in table 1 and the relative frequency of each raw material in each context is presented in

(1) (r)-Refused by the original author. table 3. Core and blank analysis considered the attributes present in tables 4 and 5, respectively, plus measurements (Tab. 4 and 5).

The original morphology of the core blanks followed Rapp and Hill (1998: 42) classification. Weight was measured on cobbles, pebbles and cores only. Fire cracked rocks were not considered for the metrical analysis. The traditional morphological classification of cores followed the Brézillon (1983) classification where non-prepared 


\begin{tabular}{|c|c|c|c|c|}
\hline \multicolumn{2}{|c|}{ 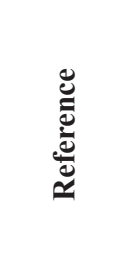 } & 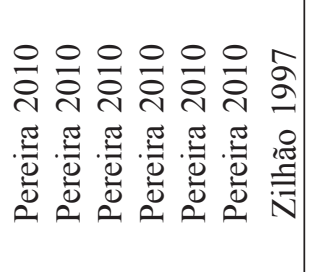 & 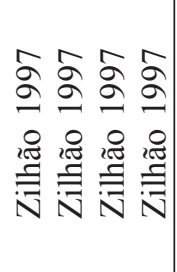 & 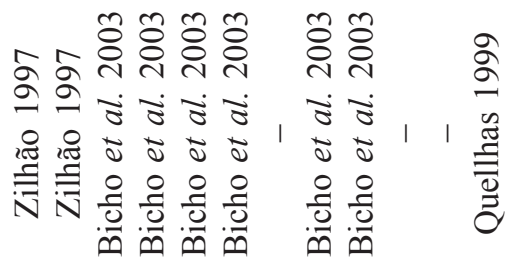 \\
\hline \multirow{5}{*}{ 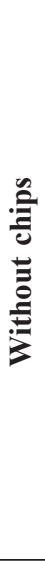 } & 茪 & 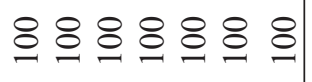 & 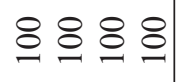 & $8 \& 88 \& 8,8 \& 1,8$ \\
\hline & $\stackrel{\bar{\Xi}}{\overline{0}}$ & 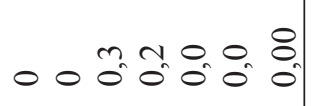 & $\begin{array}{llll}8 & 8 & n & 8 \\
0 & 0 & 0 & 0 \\
0 & 0 & 0\end{array}$ & 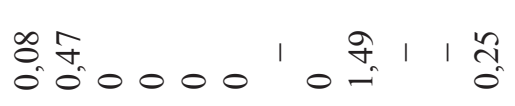 \\
\hline & 离. & 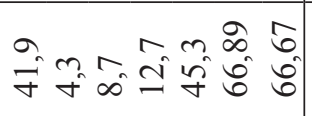 & 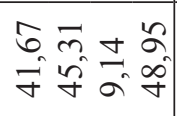 & 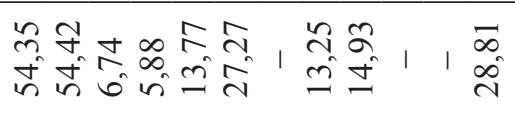 \\
\hline & 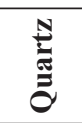 & 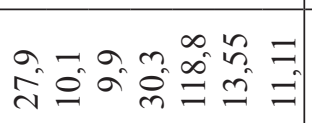 & 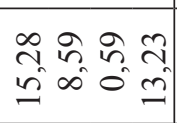 & 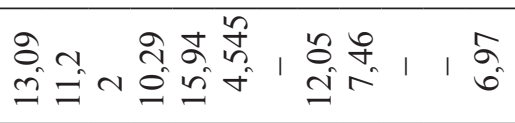 \\
\hline & 竞 & 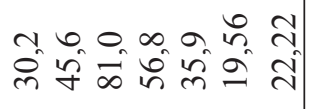 & 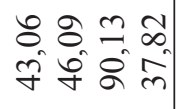 & 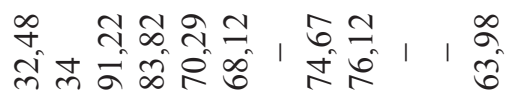 \\
\hline \multirow{5}{*}{ 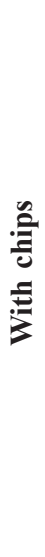 } & 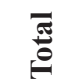 & \& \& \& \& \& & ○ & 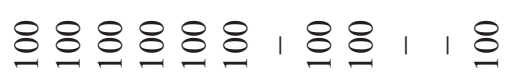 \\
\hline & 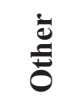 & 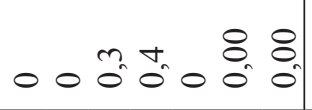 & $\begin{array}{llll}8 & 8 & 0 & 8 \\
0 & 0 & 0 & 0 \\
0 & 0 & \end{array}$ & 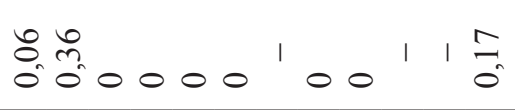 \\
\hline & 育 & 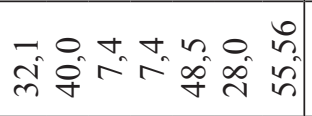 & 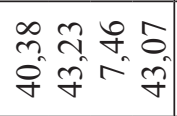 & 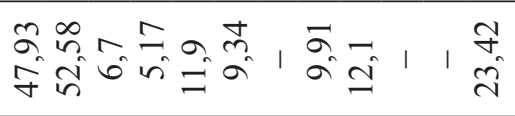 \\
\hline & 齐 & 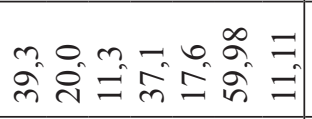 & 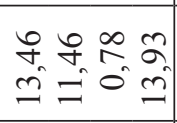 & 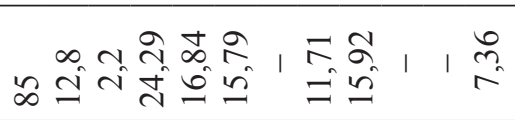 \\
\hline & $\overrightarrow{\underline{\underline{I}}}$ & 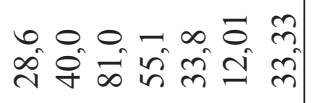 & 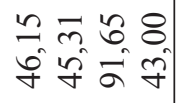 & 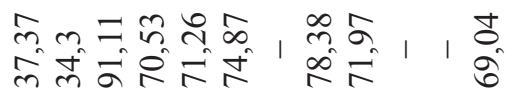 \\
\hline & $-\quad I$ & 圼层 & 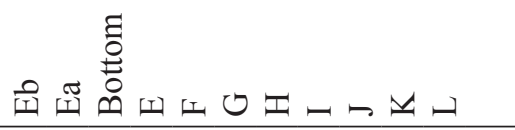 \\
\hline & 总 & 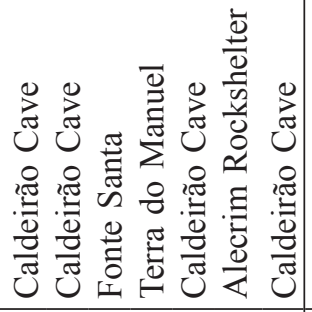 & 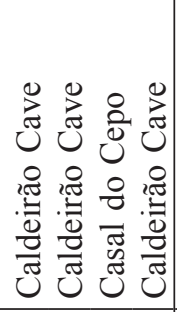 & 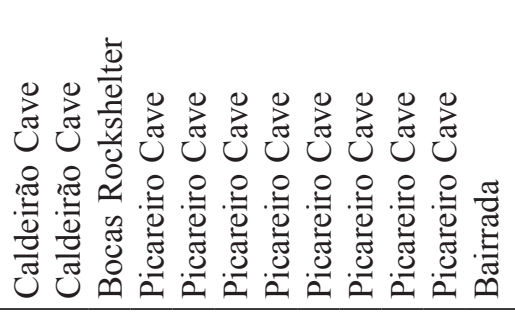 \\
\hline & 苞 & 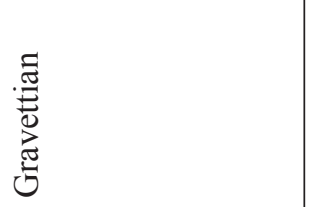 & 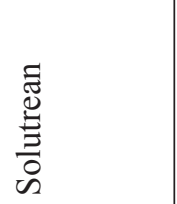 & 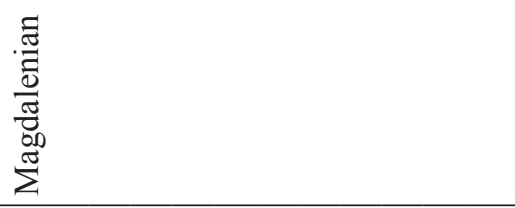 \\
\hline
\end{tabular}




\begin{tabular}{|c|c|c|c|c|}
\hline & & Gravettian & Solutrean & Magdalenian \\
\hline Concept & $\begin{array}{l}\text { Extensive } \\
\text { Stepped } \\
\text { Prismatic } \\
\text { Centripetal } \\
\text { Random } \\
\text { Poliedric } \\
\text { Fragment }\end{array}$ & $\begin{array}{r}7,7 \\
54,9 \\
17,6 \\
16,5 \\
1,1 \\
0 \\
2,2\end{array}$ & $\begin{array}{c}0 \\
38,9 \\
41,7 \\
11,1 \\
8,3 \\
0 \\
0\end{array}$ & $\begin{array}{c}0 \\
41,7 \\
34,8 \\
15 \\
4,8 \\
2,7 \\
1,1\end{array}$ \\
\hline Suport & $\begin{array}{l}\text { Slabe } \\
\text { Pebble/Cobble } \\
\text { Flake } \\
\text { Fragment }\end{array}$ & $\begin{array}{r}1,09 \\
71,74 \\
16,30 \\
1,09\end{array}$ & $\begin{array}{r}2,70 \\
48,65 \\
21,62 \\
8,11\end{array}$ & $\begin{array}{r}1,45 \\
66,67 \\
11,59 \\
9,42\end{array}$ \\
\hline Esphericity & $\begin{array}{l}\text { Sub-discoidal } \\
\text { Esferic } \\
\text { Sub-prismoidal } \\
\text { Prismoidal } \\
\text { Sub-prismoidal } \\
\text { Unrecognizable }\end{array}$ & $\begin{array}{r}4,40 \\
14,29 \\
2,20 \\
15,38 \\
15,38 \\
48,35\end{array}$ & $\begin{array}{r}5,41 \\
8,11 \\
0,00 \\
10,81 \\
27,03 \\
48,65\end{array}$ & $\begin{array}{r}2,19 \\
15,33 \\
2,92 \\
12,41 \\
30,66 \\
36,50\end{array}$ \\
\hline Rollement & $\begin{array}{l}\text { Very angular } \\
\text { Angular } \\
\text { Sub-angular } \\
\text { Sub-roled } \\
\text { Roled } \\
\text { Well rolled } \\
\text { Unrecognizable }\end{array}$ & $\begin{array}{r}1,10 \\
1,10 \\
18,68 \\
17,58 \\
20,88 \\
6,59 \\
34,07\end{array}$ & $\begin{array}{r}0,00 \\
8,11 \\
16,22 \\
8,11 \\
13,51 \\
8,11 \\
31,39\end{array}$ & $\begin{array}{r}0,73 \\
7,30 \\
4,38 \\
12,41 \\
30,66 \\
13,14 \\
45,95\end{array}$ \\
\hline Platform & $\begin{array}{l}\text { Cortical } \\
\text { Flat } \\
\text { Prepared } \\
\text { Mix }\end{array}$ & $\begin{array}{r}0,00 \\
69,23 \\
23,08 \\
2,20\end{array}$ & $\begin{array}{r}0,00 \\
70,27 \\
21,62 \\
5,41\end{array}$ & $\begin{array}{r}0,74 \\
66,18 \\
17,65 \\
6,62\end{array}$ \\
\hline Orientation & $\begin{array}{l}\text { On thickness } \\
\text { On surface } \\
\text { Combined } \\
\text { Unrecognizable }\end{array}$ & $\begin{array}{r}75,82 \\
17,58 \\
3,30 \\
3,30\end{array}$ & $\begin{array}{r}78,38 \\
18,92 \\
0,00 \\
2,70\end{array}$ & $\begin{array}{r}68,38 \\
25,00 \\
3,68 \\
2,94\end{array}$ \\
\hline Overhang & $\begin{array}{l}\text { Rect } \\
\text { Acute } \\
\text { Very acute } \\
\text { Unknwon }\end{array}$ & $\begin{array}{r}0,00 \\
71,43 \\
17,58 \\
10,99\end{array}$ & $\begin{array}{r}0,00 \\
64,86 \\
27,03 \\
8,11\end{array}$ & $\begin{array}{r}0,74 \\
61,03 \\
30,15 \\
8,09\end{array}$ \\
\hline Negatives & $\begin{array}{l}\text { Parallel unidireccional } \\
\text { Parallel bidireccional } \\
\text { Perpendicular } \\
\text { Crossed } \\
\text { Multidireccional } \\
\text { Convergent } \\
\text { Single detachment } \\
\text { Unknown }\end{array}$ & $\begin{array}{r}71,43 \\
3,30 \\
3,30 \\
2,20 \\
0,00 \\
15,38 \\
2,20 \\
2,20\end{array}$ & $\begin{array}{r}75,68 \\
0,00 \\
0,00 \\
0,00 \\
2,70 \\
13,51 \\
2,70 \\
0,00\end{array}$ & $\begin{array}{r}72,79 \\
0,00 \\
0,00 \\
2,94 \\
0,00 \\
16,91 \\
4,41 \\
0,00\end{array}$ \\
\hline
\end{tabular}

Tab. 4. Attribute analysis of the cores from the sites studied. 


\begin{tabular}{|c|c|c|c|c|}
\hline & & Gravettian & Solutrean & Magdalenian \\
\hline Cortex & $\begin{array}{l}0 \\
1-25 \\
25-50 \\
50-75 \\
75-99 \\
100\end{array}$ & $\begin{array}{r}56,5 \\
16,6 \\
7,6 \\
4,0 \\
5,3 \\
10,0\end{array}$ & $\begin{array}{r}61,6 \\
13,5 \\
7,1 \\
4,7 \\
5,6 \\
7,4\end{array}$ & $\begin{array}{r}52,7 \\
19,1 \\
10,1 \\
5,2 \\
6,3 \\
6,8\end{array}$ \\
\hline Cortex location & $\begin{array}{l}\text { Absent } \\
\text { Proximal } \\
\text { Distal } \\
\text { Lateral } \\
\text { Central } \\
\text { Orange slice } \\
\text { Sausage slice } \\
\text { Total }\end{array}$ & $\begin{array}{r}56,7 \\
0,7 \\
5,9 \\
16,5 \\
0,4 \\
9,4 \\
0,5 \\
10,0\end{array}$ & $\begin{array}{r}61,6 \\
0,5 \\
5,9 \\
14,4 \\
0,7 \\
9,4 \\
0,5 \\
7,4\end{array}$ & $\begin{array}{r}52,7 \\
1,0 \\
7,3 \\
19,5 \\
1,0 \\
11,4 \\
0,4 \\
6,8\end{array}$ \\
\hline Dorsal pattern & $\begin{array}{l}\text { Cortical } \\
\text { Unidirectional parallel } \\
\text { Unidirectional convergent } \\
\text { Unidirectional opposed } \\
\text { Bidireccional } \\
\text { Crossed } \\
\text { Centripetal } \\
\text { Multidireccional } \\
\text { Unknown } \\
\text { Bulb }\end{array}$ & $\begin{array}{r}10,8 \\
58,7 \\
15,4 \\
0,8 \\
1,7 \\
8,9 \\
1,3 \\
0,2 \\
2,2 \\
0,0\end{array}$ & $\begin{array}{r}8,4 \\
62,7 \\
14,8 \\
1,1 \\
2,4 \\
9,4 \\
0,5 \\
0,1 \\
0,7 \\
8,4\end{array}$ & $\begin{array}{r}6,5 \\
67,3 \\
12,2 \\
1,1 \\
1,4 \\
8,7 \\
1,2 \\
0,05 \\
1,5 \\
6,5\end{array}$ \\
\hline Axis & $\begin{array}{l}\text { On axis } \\
\text { Of axis } \\
\end{array}$ & $\begin{array}{l}54,5 \\
45,5\end{array}$ & $\begin{array}{l}47,8 \\
52,2\end{array}$ & $\begin{array}{l}76,9 \\
23,1\end{array}$ \\
\hline Edges & \begin{tabular}{|l|} 
Parallel \\
Convergent \\
Divergent \\
Biconvexe \\
Concave-convexe \\
Biconcave \\
Irregular \\
\end{tabular} & $\begin{array}{r}16,2 \\
21,0 \\
25,6 \\
5,9 \\
0,6 \\
4,2 \\
26,5\end{array}$ & $\begin{array}{r}17,0 \\
16,0 \\
22,8 \\
5,1 \\
0,5 \\
6,1 \\
32,6\end{array}$ & $\begin{array}{r}17,9 \\
16,8 \\
24,2 \\
7,5 \\
5,3 \\
0,3 \\
27,9\end{array}$ \\
\hline Profile & \begin{tabular}{|l} 
Flat \\
Concave \\
Twisted \\
\end{tabular} & $\begin{array}{r}92,9 \\
5,3 \\
1,8 \\
\end{array}$ & $\begin{array}{r}91,4 \\
6,6 \\
2,0 \\
\end{array}$ & $\begin{array}{r}91,9 \\
6,1 \\
2,0 \\
\end{array}$ \\
\hline Section & $\begin{array}{l}\text { Flat } \\
\text { Triangular } \\
\text { Trapezoidal } \\
\text { Irregular }\end{array}$ & $\begin{array}{l}31,4 \\
34,1 \\
23,5 \\
11,0\end{array}$ & $\begin{array}{l}35,0 \\
33,1 \\
21,8 \\
10,0\end{array}$ & $\begin{array}{l}29,1 \\
36,1 \\
22,5 \\
12,4\end{array}$ \\
\hline End & $\begin{array}{l}\text { Thick } \\
\text { Feathered } \\
\text { Stepp } \\
\text { Overpass } \\
\text { Pointed } \\
\text { Retouched } \\
\text { Fracture } \\
\end{array}$ & $\begin{array}{r}6,0 \\
46,5 \\
29,1 \\
2,6 \\
4,6 \\
1,7 \\
9,5 \\
\end{array}$ & $\begin{array}{r}3,5 \\
40,6 \\
33,5 \\
4,0 \\
6,1 \\
0,6 \\
11,2 \\
\end{array}$ & $\begin{array}{r}5,3 \\
39,0 \\
27,5 \\
3,2 \\
8,2 \\
2,6 \\
13,8 \\
\end{array}$ \\
\hline Bulb & $\begin{array}{l}\text { Pronounced } \\
\text { Difuse } \\
\text { Pronounced chipped } \\
\text { Difuse chipped } \\
\text { Compete chipped } \\
\text { Double not chipped } \\
\text { Double chipped }\end{array}$ & $\begin{array}{r}28,9 \\
42,8 \\
4,4 \\
6,1 \\
0,6 \\
0,7 \\
0,2\end{array}$ & $\begin{array}{r}34,1 \\
28,5 \\
14,4 \\
6,7 \\
1,6 \\
0,7 \\
34,1\end{array}$ & $\begin{array}{r}28,5 \\
39,3 \\
8,8 \\
6,3 \\
1,4 \\
1,0 \\
0,4\end{array}$ \\
\hline
\end{tabular}

Tab. 5. Attribute analysis of the flakes from the sites studied (continued on next page). 


\begin{tabular}{l|l|c|c|c} 
& & Gravettian & Solutrean & Magdalenian \\
\hline Lipping & Present & 57,6 & 41,7 & 65,0 \\
& Absent & 12,7 & 12,6 & 20,8 \\
\hline Butt & Cortical & 63,8 & 61,5 & 57,8 \\
& Flat & 13,5 & 16,8 & 16,0 \\
& Dihedral & 1,7 & 0,7 & 2,2 \\
Faceted & 0,8 & 1,3 & 1,5 \\
& Winged & 0,1 & 0,5 & 1,6 \\
& Liear & 3,2 & 4,0 & 1,2 \\
& Punctiform & 0,8 & 1,4 & 1,7 \\
& Smashed & 0,7 & 0,8 & 0,1 \\
& Retouched & 0,1 & 13,1 & \\
\hline
\end{tabular}

Tab. 5. Attribute analysis of the flakes from the sites studied (continuation).

cores on cobbles and pebbles intended for the production of flakes were classified as choppers. To avoid this simplistic classification, we adapted the approach by Benito del Rey and Benito Álvarez (1998: 64-108). Cores were first divided into extensive, intensive and pre-determinate exploitation categories. Extensive exploitation targeted the production of big flakes or fragments that were after used as cores or massive tools. Intensive exploitation is related to the production of many small and pre-determined flakes as described by Inizan et al. (1999: 61). 'Stepped cores' are those that in François Bordes typology would fit-in the category of chopper. The non-use of the term chopper results of the fact that they are cores and not retouched tools. For the typological classification of retouched tools, we used the most common type-list for the Upper Paleolithic (SonnevilleBordes and Perrot 1954, 1955, 1956), which has been adapted for the Portuguese territory (Zilhão 1997). Since the main object of this study was the quartzite macrolithic component, we combined Sonneville-Bordes and Perrot with the type-list for the Lower and Middle Paleolithic (Bordes 1961) (Tab. 6).

This option allowed a more detailed description of the tool components, especially concerning the variation within sidescrapers. Intensive refitting was performed in all assemblages, sometimes with considerable results (Fig. 2), which reinforced the results from attribute analysis.

As complementary information, it has to be said that orthoquartzite and metaquartzite can be found in the same secondary deposits. Both represent a compact and hard rock formed by fine grains of strongly united quartz that fractures conchoidally or sub-conchoidally through the grains rather than around them (Costa 1998; Farndon 2006). Since they represent a very distinct raw material when compared to flint or quartz, we will refer to both simply as quartzite. No microscopic study was carried out to separate the quartzite granulometry.

\section{RESULTS}

The description of the results is illustrated by the tables, figures and graphics. Pebbles and cobbles are water-worn and cortex represents a very thin cap as a regular surface, which do not seem to influence the impact of hammer in the platform of percussion or quality of the cutting edges. That seems to be corroborated by the absence of preparation of the cores, including their configuration. This resulted in the predominance of cortical butts and sticking platforms. There are, however, some cases of maintenance, namely, the removal of problems such as the irregularity of the flaking surface front resulting from stepped detachments or pronounced irregularities in the overhang (Fig. 3).

Six different reduction strategies were identified (Tab. 4). The Extensive concept seems to have been intended to split big round cobbles, often weighing between 2 and $4 \mathrm{Kg}$. The resulting big and thick flakes, the remaining core and, sometimes, bigger fragments that resulted from this knapping were subsequently used as base for the recurrent obtainment of flakes that fall into the standard dimensions. 


\begin{tabular}{|c|c|c|c|c|c|c|c|c|}
\hline & \multicolumn{2}{|c|}{ Gravettian } & \multicolumn{2}{|c|}{ Solutrean } & \multicolumn{2}{|c|}{ Magdalenian } & \multicolumn{2}{|c|}{ Total } \\
\hline & $\#$ & $\%$ & $\#$ & $\%$ & $\#$ & $\%$ & $\#$ & $\%$ \\
\hline Endscraper: typical in flake & & & & & 3 & 0,8 & 3 & 0,6 \\
\hline Endscraper: atypical in flake & & & & & 1 & 0,3 & 1 & 0,2 \\
\hline Endscraper: in retouched flake & & & & & 2 & 0,5 & 2 & 0,4 \\
\hline Endscraper: typical carinated & 1 & 1,0 & & & 2 & 0,5 & 3 & 0,6 \\
\hline Endscraper: atypical carinated & 1 & 1,0 & & & 1 & 0,3 & 2 & 0,4 \\
\hline Endscraper: nosed & & & & & 1 & 0,3 & 1 & 0,2 \\
\hline Endscraper coreshapped & & & & & 1 & 0,3 & 1 & 0,2 \\
\hline Rabot & 1 & 1,0 & & & 5 & 1,3 & 6 & 1,1 \\
\hline Burin-truncation & & & & & 1 & 0,3 & 1 & 0,2 \\
\hline Perforator: typical & 2 & 2,0 & & & 2 & 0,5 & 4 & 0,8 \\
\hline Perforator: atypical & & & 1 & 2,2 & & & 1 & 0,2 \\
\hline Burin: dihedral straight & 2 & 2,0 & 1 & 2,2 & 5 & 1,3 & 8 & 1,5 \\
\hline Burin: dihedral dejete & & & & & 3 & 0,8 & 3 & 0,6 \\
\hline Burin: dihedral angled & & & & & 2 & 0,5 & 2 & 0,4 \\
\hline Burin: dihedral transversal on lateral truncation & & & & & 1 & 0,3 & 1 & 0,2 \\
\hline Truncation: oblique on flake & & & & & 1 & 0,3 & 1 & 0,2 \\
\hline Blade: retouched & & & 1 & 2,2 & 1 & 0,3 & 2 & 0,4 \\
\hline Blade: both sides retouched & & & & & 1 & 0,3 & 1 & 0,2 \\
\hline Atypical point & 1 & 1,0 & & & & & 1 & 0,2 \\
\hline Vale Comprido Point & 2 & 2,0 & & & 4 & 1,1 & 5 & 1,0 \\
\hline Pic & 1 & 1,0 & & & 1 & 0,3 & 2 & 0,4 \\
\hline Notch & 22 & 21,8 & 11 & 23,9 & 79 & 20,9 & 112 & 21,5 \\
\hline Distal notch & & 0,0 & & & 4 & 1,1 & 4 & 0,8 \\
\hline Denticulate & 16 & 15,8 & 4 & 8,7 & 50 & 13,2 & 70 & 13,4 \\
\hline Sidescrapper: simple straight & 3 & 3,0 & 2 & 4,3 & 12 & 3,2 & 17 & 3,3 \\
\hline Sidescrapper: simple convexe & 3 & 3,0 & 3 & 6,5 & 14 & 3,7 & 20 & 3,8 \\
\hline Sidescrapper: simple concave & 1 & 1,0 & & & 6 & 1 , & 7 & 1,3 \\
\hline Sidescrapper: doble straight & 1 & 1,0 & 1 & 2,2 & 1 & 0,3 & 3 & 0,6 \\
\hline Sidescrapper: doble straight-convexe & & & & & 2 & 0 & 2 & 0,4 \\
\hline Sidescrapper: doble biconvexe & & & & & 1 & 0,3 & 1 & 0,2 \\
\hline Sidescrapper: doble straight biconcave & & & 2 & 4,3 & 2 & 0,5 & 2 & 0,4 \\
\hline Sidescrapper: doble straight concave-convexe & & & & & 3 & 0,8 & 3 & 0,6 \\
\hline Sidescrapper: convergent straight & & & & & 2 & 0,5 & 2 & 0,4 \\
\hline Sidescrapper: convergent convexe & & & & & 3 & 0,8 & 3 & 0,6 \\
\hline Sidescrapper: dejete & & & & & 1 & 0,3 & 1 & 0,2 \\
\hline Sidescrapper: transversal straight & 1 & 1,0 & & & 5 & 1,3 & 6 & 1,1 \\
\hline Sidescrapper: transversal convexe & 1 & 1,0 & & & 2 & 0,5 & 3 & 0,6 \\
\hline Sidescrapper: transversal concave & & & & & 1 & 0,3 & 1 & 0,2 \\
\hline Sidescrapper: transversal on flat face & & & & & 2 & 0,5 & 2 & 0,4 \\
\hline Sidescrapper: with abrupt retouch & 2 & 2,0 & & & 1 & 0,3 & 3 & 0,6 \\
\hline Sidescrapper: bifacial retouch & 1 & 1,0 & & & & & 1 & 0,2 \\
\hline Retouched flake or fragment & 8 & 7,9 & 12 & 26,1 & 61 & 16,1 & 81 & 15,5 \\
\hline Flake with atypical retouch & & & & & 3 & 0,8 & 3 & 0,6 \\
\hline Very big retouched flake & & & & & 1 & 0,3 & 1 & 0,2 \\
\hline Flake with retouch on the ventral face & & & & & 1 & 0,3 & 1 & 0,2 \\
\hline Chopper/chopping-tool & 9 & 8,9 & & & 26 & 6,9 & 35 & 6,7 \\
\hline Chopper/chopping-tool: inverse & & & & & 2 & 0,5 & 2 & 0,4 \\
\hline Microburin & & & & & 1 & 0,3 & 1 & 0,2 \\
\hline Hammerstone & 20 & 19,8 & 8 & 17,4 & 38 & 10,1 & 66 & 12,6 \\
\hline Anvil & 1 & 1,0 & & & 7 & 1,9 & 8 & 1,5 \\
\hline & & & & & 5 & 1,3 & 5 & 1,0 \\
\hline Grinding stone & 1 & 1,0 & & & & & 1 & 0,2 \\
\hline Trihedral & & & & & 1 & & 1 & 0,2 \\
\hline Uniface & & & & & 2 & 0,5 & 2 & 0,4 \\
\hline Bifacial tool & & & & & 1 & 0,3 & 1 & 0,2 \\
\hline Total & 101 & 100,0 & 46 & 100,0 & 378 & 100,0 & 522 & 100,0 \\
\hline
\end{tabular}

Tab. 6. Inventory of the tools from the sites studied. 


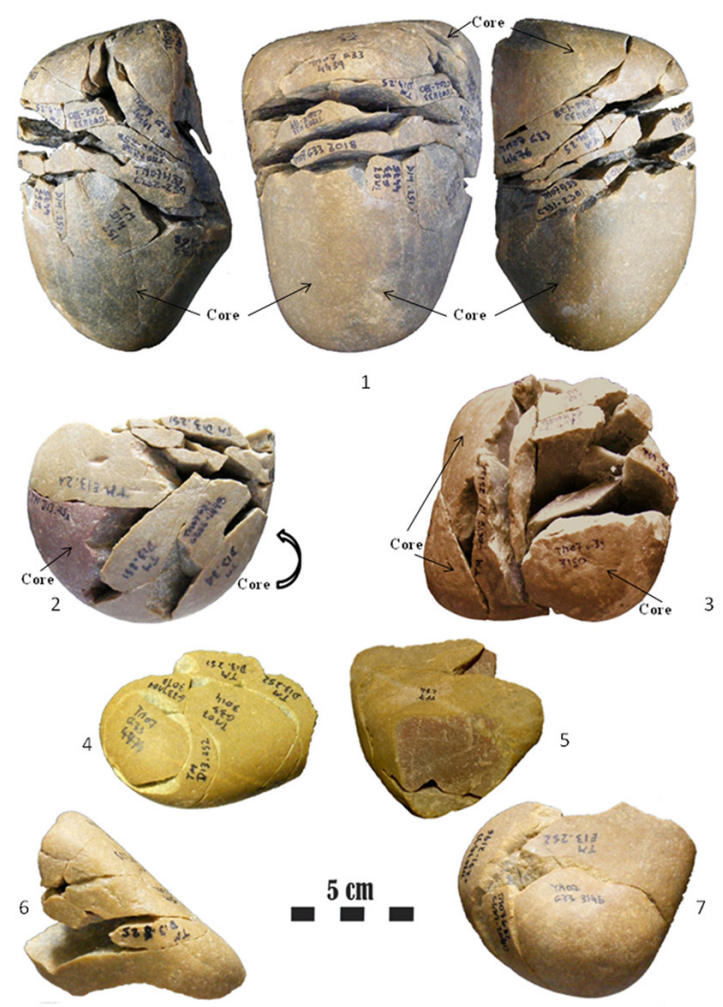

Fig. 2. Refitting on Gravettian contexts. 1. Extensive core, splintered in two cores; 2. Extensive core, splintered in two cores; 3. Extensive core, splintered in three cores; 4. Stepped reduction; 5. Stepped reduction; 6. Prismatic reduction; 7. Stepped reduction. All from Terra do Manuel (Rio Maior).
The Stepped reduction aimed the production of standard flakes according to an intensive concept. This strategy tended to start in a cortical platform, and follow the thickness of the volume. Detachments were oblique, orthogonal and sometimes, usually the last ones that failed and, therefore, definitely jeopardized the continuation of the reduction can even be obtuse. Reduction was unipolar, unifacial, unidirectional parallel or convergent sequence. The parallel or convergent orientation of the detachments depended in the platform being more or less flat. During the knapping process, these tended to lateralized progressively, especially in the pebbles that had a more discoidal shape.

Prismatic reduction was aimed for the production of flakes, blades and bladelets. It also started with cortical platforms, and followed the thickness of the volume in a unipolar, unifacial and unidirectional parallel sequence. Pebbles used as support tended to be thicker and more angular than those from the Stepped examples. Platforms also tended to be flatter and more regular. Reduction usually started in a natural crest or, in other words, a natural apex of the block. A perpendicular platform that creates crossed detachments may be present in the final moments of exploitation of the blocks, with negatives usually overlapping but rarely alternating with the prior. This situation represents a rotation of the core in order to follow the same strategy in a new platform and not a
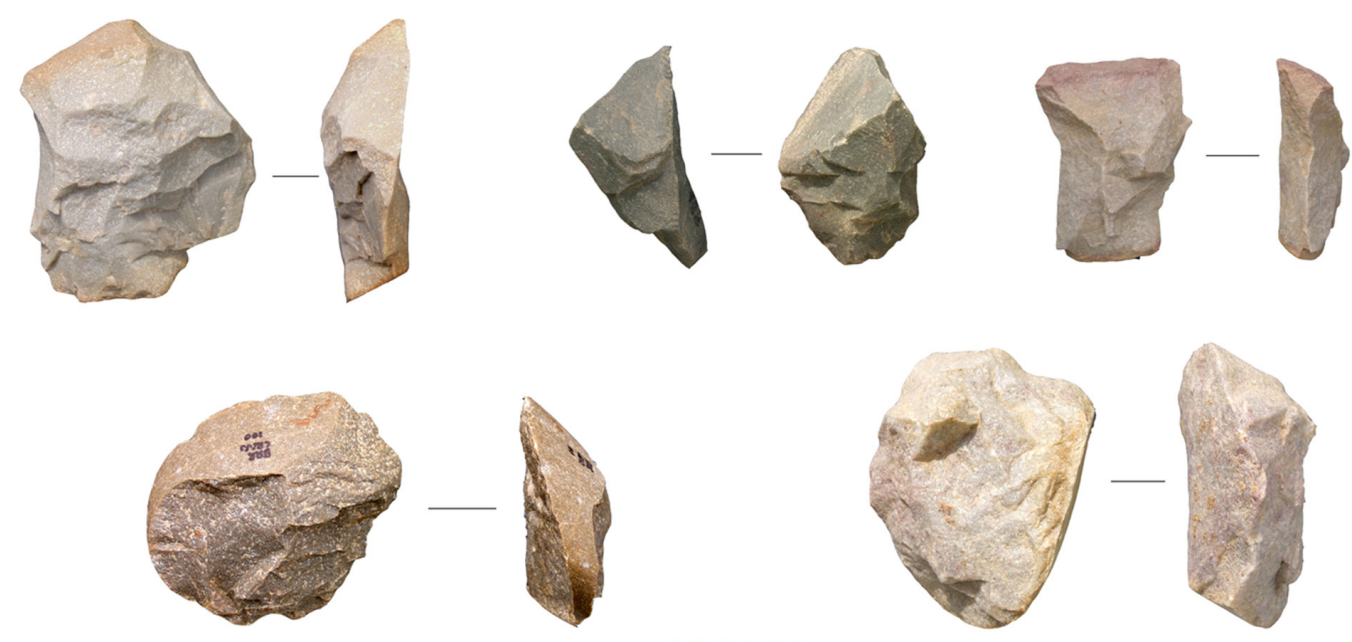

\section{$5 \mathrm{~cm}$}

Fig. 3. Maintenance flakes. All from Bairrada (Torres Novas). 


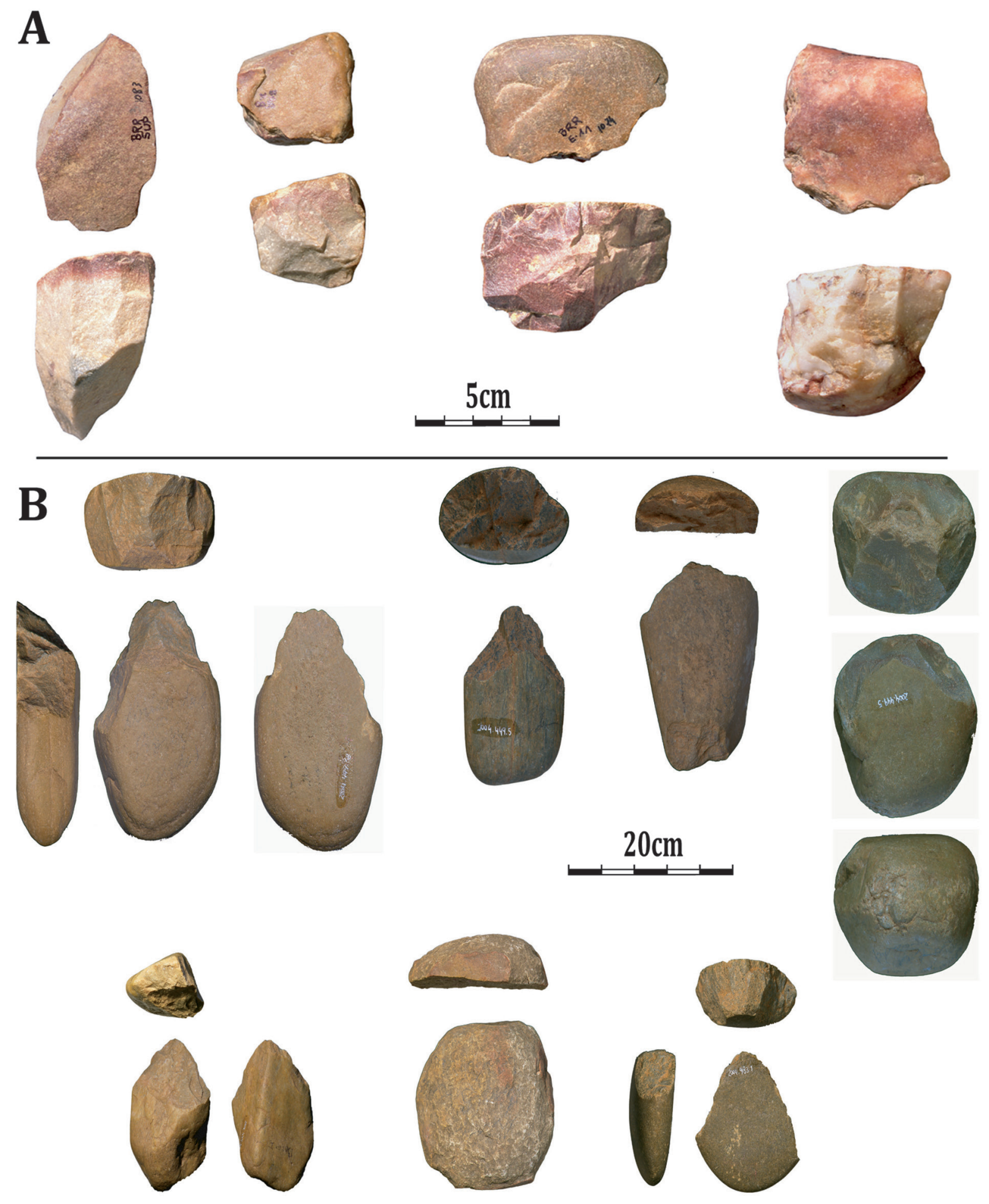

Fig. 4. A. Prismatic cores from Bairrada (Torres Novas); B. Massive tools from Bocas Rockshelter (Rio Maior). 
deliberate variation of the prismatic concept with alternate or alternant crossed detachments.

The production of small flakes was the objective of the Centripetal reduction. The supports for the development of this concept were thick flakes or fragments, often resulting from the division of the big cobbles. It is rarely present in small pebbles. Some bigger cores can be recognized during the Gravettian, usually related with the exploitation of the flakes or fragments that resulted from the sub-division of big cobbles. Nevertheless, they tend to be rather smaller than the stepped and prismatic ones. In the case of flake supports, the detachments tended to be concentrated on the bulb. That is not surprising, since this is the place on the flake where exists a higher quantity of mass available. Due to the location of the detachments, some of these cores where the detachments are more concentrated in the bulb and not so much on the perimeter of the flake, can fall in the category of Kombewa cores. The goal was to reduce the volume's surface, through centripetal unifacial or, less frequently, bifacial detachments.

Based on their frequency, one can say that the other concepts, such as the Random and Polyhedral ones, can be considered as result of punctual circumstances that demanded a better use of volumes of raw material, often, big fragments or cobbles with major imperfections. During the Gravettian, the initial moments of the stepped reduction could have been slightly different, starting in one of the most angular corners of the initial blank, with the detachment of several overlapping flakes, sometimes alternating with others struck from the top of the front of débitage in use. During this phase, the number of flakes with cortical and flat butts can be even; however, before this area is exhausted, the exploitation expands laterally, following a standard unipolar unifacial parallel sequence. The recognition of this variance was only possible through refitting (Fig. 2: 5). Extensive cores to produce smaller cores, resulting in a high core to cobble ratio, are only present during this period (Fig. 2: 1-3). During the Solutrean the blanks used for the cores were always pebbles with spherical or sub-prismoidal shapes, but never cobbles and the reduction sequences remained relatively stable, starting in the most suitable sector and progressively reducing it to exhaustion. Stepped reduction rarely presented any kind of variation. The Centripetal cores were much smaller and, sometimes, made in quartzite similar to that found in the Solutrean pre-determined flakes (see below). During the Magdalenian, the cobbles/pebbles chosen to serve as cores tended to be as large as those from the Gravettian but were more angular, with a wide and flat platform. The Prismatic exploitation usually started along a natural well marked crest. The intention of the Magdalenian knapper to produce elongated blanks is clear, and not circumstantial as in the previous phase (Fig. 4A). Massive tools, sometimes in the shape of big thick flakes with strong distal cutting-edges, often presenting edge damage are common (Fig. 4B). The Centripetal reduction is usually evident on very small cores, on very fine quartzite (Fig. 5).

A diachronic comparison of the core frequency (Fig. 6) shows that during Gravettian compounds are clearly dominated by the Stepped reduction $(53.8 \%)$, followed from far by the Prismatic (18.3\%) and Centripetal (17.2\%). Random strategies are rare. During the Solutrean, cores assemblages are evenly dominated by Prismatic and Stepped reductions (41.2\% each), while the frequency of Centripetal as well as Random/ Polyhedral is low $(8.8 \%$ each). Finally, in the Magdalenian core assemblage, Prismatic reduction takes the lead $(41.4 \%)$, followed by the Stepped $(37.6 \%)$, from far by the Centripetal

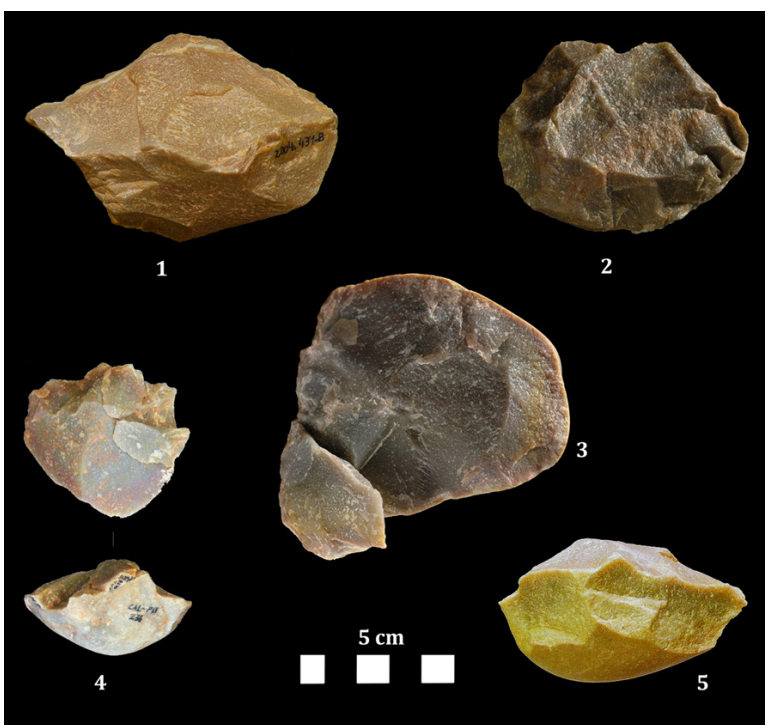

Fig. 5. Centripetal cores: 1. Bocas Rockshelter (Rio Maior); 2 and 3. Alecrim Rockshelter (Lapedo); 4. Caldeirão Cave (Tomar); 5. Terra do Manuel (Rio Maior). 

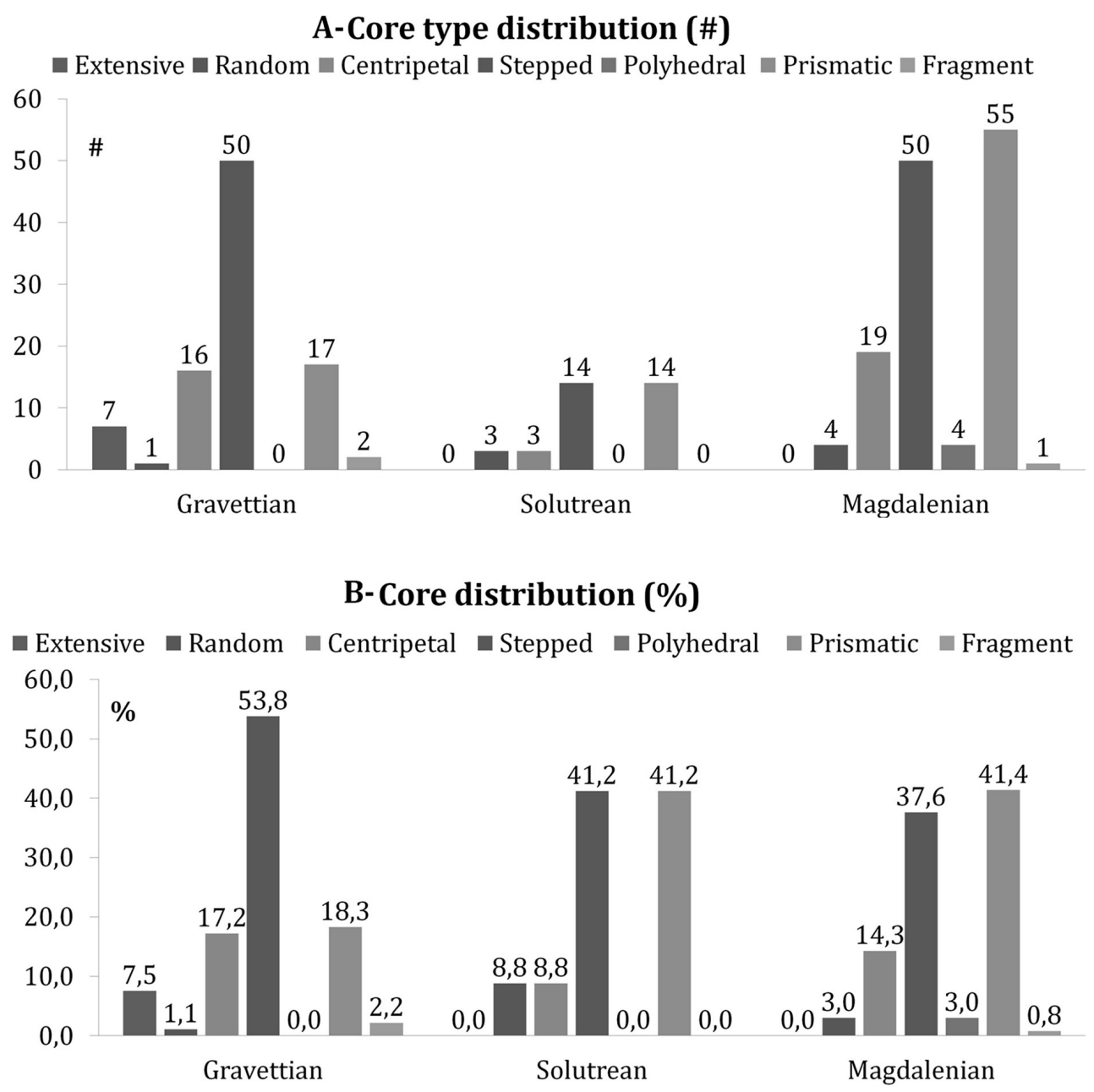

Fig. 6. Quartzite exploitation during the Upper Paleolithic of Southwest Iberian Peninsula: A. Diachronic variability of cores in number; B. Diachronic variability of cores in percentage.

$(14.3 \%)$. Polyhedral and Random cores are rare $(3 \%)$.

The diachronic analysis of the relative frequency of core types shows that from Gravettian to Magdalenian the Stepped reduction decreases, the Prismatic increases, and these two are the ones that always dominate the assemblages (Fig. 6B). The curve from the Centripetal cores show a considerable reduction during the Solutrean and seem to have a response from the one of Random cores. Proportional response seems to occur also between the Extensive and Polyhedral, despite the different objectives of each concept.

The $\chi^{2}$ test on the null hypothesis of significant chronological difference between core assem- blages indicate that Gravettian and Solutrean as Gravettian and Magdalenian are significantly different, but Solutrean and Magdalenian are not (Tab. 7). This is corroborated with the Cluster analysis, based on the Euclidean distance between groups (Fig. 7A). This fact might be related with the considerable low amount of cores in the Solutrean and, therefore, should be considered with caution. First, because it concerns only the typological variability; Second, and more importantly, because it is not corroborated by the same tests performed on the technological data obtained from the core assemblages. In fact, the $\chi^{2}$ test (Tab. 8) and cluster analysis (Fig. 7B) on the technologic attributes performed on the core assemblages clusters Gravettian with Mag- 


\begin{tabular}{|l|c|c|c|}
\hline \multicolumn{3}{|c|}{ Cores-Typology } \\
\hline & Gravettian vs. Solutrean & Gravettian vs. Magdalenian & Gravettian vs. Magdalenian \\
\hline N1: & 96 & 98 & 96 \\
N2: & 98 & 98 & 98 \\
Degree of freedom: & 4 & 4 & 5 \\
Chi^2: & 26,756 & 7,321 & 23,504 \\
p(same): & $2,23 \mathrm{E}-05$ & 0,11987 & 0,00027035 \\
Monte Carlo p(same): & $<0,0001$ & 0,1151 & 0,0001 \\
Fisher exact $p($ same): & N/A & N/A & N/A \\
\hline
\end{tabular}

Tab. 7. Chi2 analysis on the typologic features of the core assemblages.

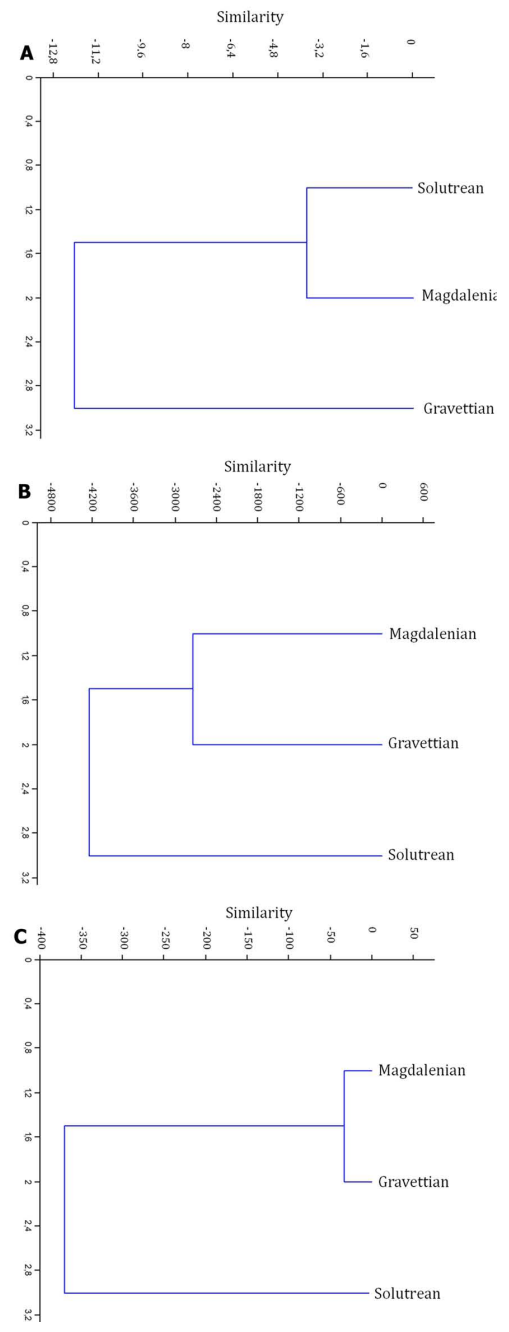

Fig. 7. Quartzite exploitation during the Upper Paleolithic of Southwest Iberian Peninsula. Cluster analysis based on the Euclidean distance between groups using: A. Cores: Typologic criteria; B. Cores: Technologic criteria; C. Entire assemblages: after pebbles, fragments, chips and firecracked rocks were excluded. dalenian, meaning that these two are technologically more similar (e.g. have more in common) between them than with Solutrean. Finally, because the typological similarity and clustering of the core assemblage is not corroborated by the same $\chi^{2}$ test performed on the entire assemblages (Tab. 9), nor even by the same the $\chi^{2}$ test (Tab. 10) or cluster analysis (Fig. 7c) performed on the assemblages after pebbles, fragments, chips and firecracked rocks were excluded. This likeness between Gravettian and Magdalenian quartzite assemblages is not a surprise, once it follows the pattern already known for the flint and quartz.

The combination of technological analysis with refitting shows that quartzite exploitation intended to produce flakes, which always represent more than $92 \%$ of the blank assemblage. A progressive but still slight increase in the number of bladelets exists towards the Madgalenian as there is a slight peak in the frequency of blades during the Solutrean (Fig. 8).

The attribute analysis on the flakes seems to show a general standardization during the three periods (Tab. 5). The majority of the flakes do not present cortex on the dorsal face and only a few have more than $50 \%$ of cortex. This element is often located along a lateral edge and present mostly in the bigger flakes. Unidirectional parallel scars dominate the dorsal patterns and tend to increase in time by opposite to cortical and unidirectional convergent. On-axis detachments increase considerably during the Magdalenian. Flat profiles are slightly more abundant during the Solutrean, while triangular are slightly more abundant during the Gravettian and the Magdalenian. Feathered ends decrease from Gravettian to Magdalenian, overpass and fractured in- 


\begin{tabular}{|l|c|c|c|}
\hline \multicolumn{4}{|c|}{ Gravettian vs. Solutrean } \\
\hline \multicolumn{1}{|c|}{ One constraint } & Gravettian vs. Solutrean & Solutrean vs. Magdalenian & Gravettian vs. Magdalenian \\
\hline N1: & 71,145 & 61,219 & 71,145 \\
N2: & 61,219 & 62,847 & 62,847 \\
Degree of freedom: & 85 & 84 & 85 \\
Chi^2: & 69,328 & 59,715 & 27,426 \\
p(same): & 0 & 0 & 0 \\
Monte Carlo p(same): & mplabel & mplabel & mplabel \\
Fisher exact $p($ same): & N/A & N/A & N/A \\
\hline
\end{tabular}

Tab. 8. Chi2 analysis on the technologic features of the core assemblages.

\begin{tabular}{|l|c|c|c|}
\hline \multicolumn{3}{|c|}{ Total assemblage } \\
\hline & Gravettian vs. Solutrean & Solutrean vs. Magdalenian & Gravettian vs. Magdalenian \\
\hline $\mathrm{N} 1:$ & 3,125 & 1,425 & 3,125 \\
$\mathrm{~N} 2:$ & 1,425 & 3,174 & 3,174 \\
Degree of freedom: & 8 & 8 & 8 \\
Chi^2: & 279,04 & 358,04 & 223,08 \\
p(same): & $1,18 \mathrm{E}-55$ & $1,74 \mathrm{E}-72$ & $8,61 \mathrm{E}-44$ \\
Monte Carlo $\mathrm{p}(\mathrm{same}):$ & $<0,0001$ & $<0,0001$ & $\mathrm{mplabel}$ \\
Fisher exact $\mathrm{p}($ same): & N/A & N/A & N/A \\
\hline
\end{tabular}

Tab. 9. Chi2 analysis on the assemblage variability considering all categories.

\begin{tabular}{|l|c|c|c|}
\hline \multicolumn{4}{|c|}{ Total assemblage (without pebbles, fragments, firecracks and chips) } \\
\hline & Gravettian vs. Solutrean & Solutrean vs. Magdalenian & Gravettian vs. Magdalenian \\
\hline N1: & 1,989 & 1,141 & 1,989 \\
N2: & 1,141 & 2,060 & 2,060 \\
Degree of freedom: & 4 & 4 & 4 \\
Chi^2: & 94,27 & 67,51 & 30,579 \\
p(same): & $1,63 \mathrm{E}-19$ & $7,61 \mathrm{E}-14$ & $3,73 \mathrm{E}-06$ \\
Monte Carlo $\mathrm{p}(\mathrm{same}):$ & $<0,0001$ & $<0,0001$ & $<0,0001$ \\
Fisher exact $\mathrm{p}(\mathrm{same}):$ & N/A & N/A & N/A \\
\hline
\end{tabular}

Tab. 10. Chi2 analysis on the assemblage variability excluding pebbles, fragments, firecracks and chips.

crease, while stepped are more abundant during the Solutrean. Lipping is much less abundant during Solutrean. Edge morphology varies considerably. The most common shape is the irregular (especially during the Solutrean) and divergent (especially in the Gravettian, when convergent are also very frequent). Parallel are slightly more abundant towards the Magdalenian. Biconvex along with concave-convex shapes are rare. Pro- nounced bulbs are considerably more abundant during the Solutrean than during the other two periods. As it happens with the cores, the cluster analysis performed over the results from the technological analysis grouped the Magdalenian assemblage with the Gravettian, meaning that flake assemblage from pre and post Last Glacial Maximum has more in common in between than with Solutrean or this one with any of the others 


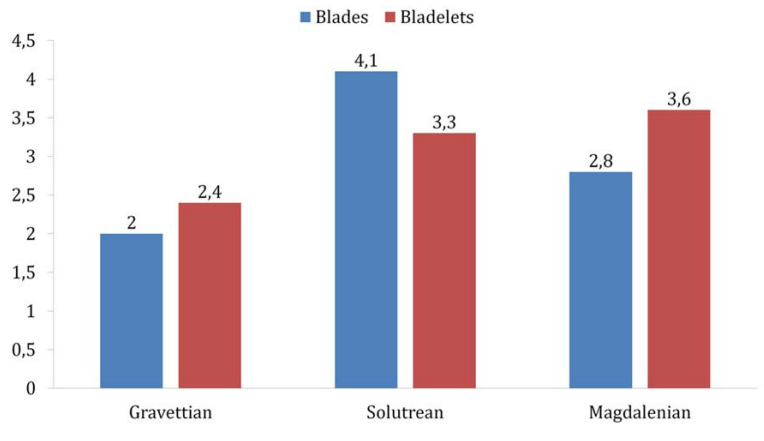

Fig. 8. Quartzite exploitation during the Upper Paleolithic of Southwest Iberian Peninsula. Diachronic frequency of blades and bladelets.

(Fig 9A); this is corroborated by the Principal Coordinates analysis (Fig. 9B).

Flakes tend to be small, usually not bigger than $40 \mathrm{~mm}$ in length and $30 \mathrm{~mm}$ in width, with the Solutrean ones slightly larger and standardized (Fig. 10A). Again, the cluster analysis on the length, width and thickness of the three flake assemblages, clusters Gravettian with Magdalenian (Fig. 10B). This result that is corroborated by the Principal Coordinates analysis (Fig. 10C).

In some Solutrean contexts, we have found a small number of flakes (with 5 to $10 \mathrm{~cm}$ length $-\sim 8 \mathrm{~cm}$ ), that are relatively thin, on-axis, with lateral sharp edges, feathered ending, and produced in types of quartzite different from those present in the remaining assemblages. None was refitted, albeit some seem to come from the same block. Their characteristics do not fit any type or individual from the core assemblages and they are clearly distinct from the remaining flake assemblages. They were not recovered in Casal do Cepo (Middle Solutrean), but were found in Caldeirão (Middle and Upper Solutrean, layers Fa, $\mathrm{Fb}$ ) and in Layer A of Vale Boi (Upper Solutrean), and in the latter case along with a fragment of Parpalló point (Fig. 11).

The quantity of tools varies considerably and, like with the cores, they are considerably fewer in the Solutrean. Typical Upper Paleolithic retouched tools are more abundant during the Magdalenian as are sidescrappers (Tab. 6). Percussion tools are very common in quartzite assemblages and can frequently occur in association with massive retouched tools such as choppers. During the Gravettian, retouched tools are dominated by
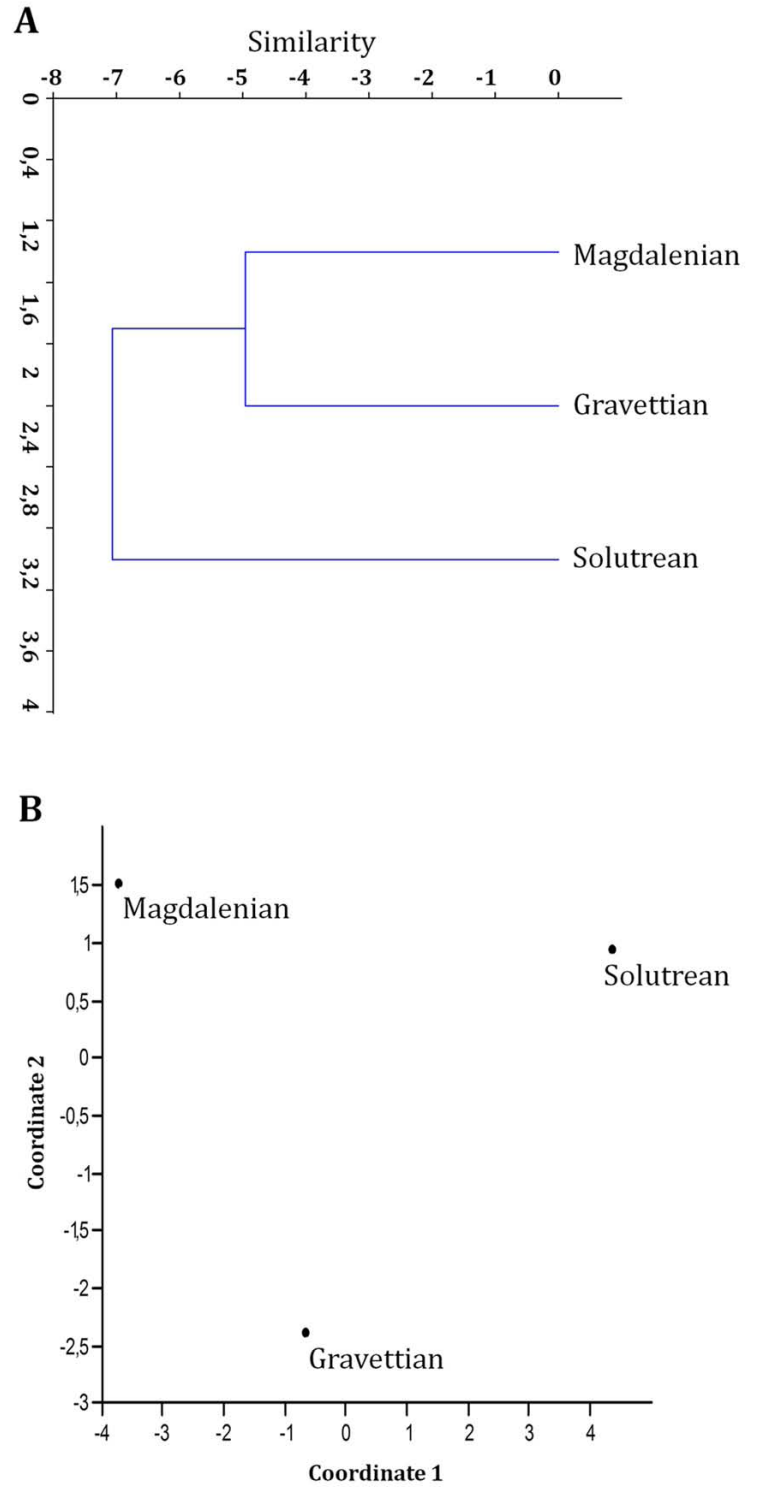

Fig. 9. Quartzite flake production during the Upper Paleolithic of Southwest Iberian Peninsula: A. Cluster analysis based on the Euclidean distance between groups using technologic criteria; B. Principal Coordinates analysis using technologic criteria.

notches and denticulates. Sidescrapers represent $12.9 \%$ of the assemblage, being the simple sidescrapper group more frequent than double and convergent. Typical Upper Paleolithic tools are very rare. During the Solutrean, choppers and chopping-tools disappear. Notches along with retouched flakes and fragments were the most com- 

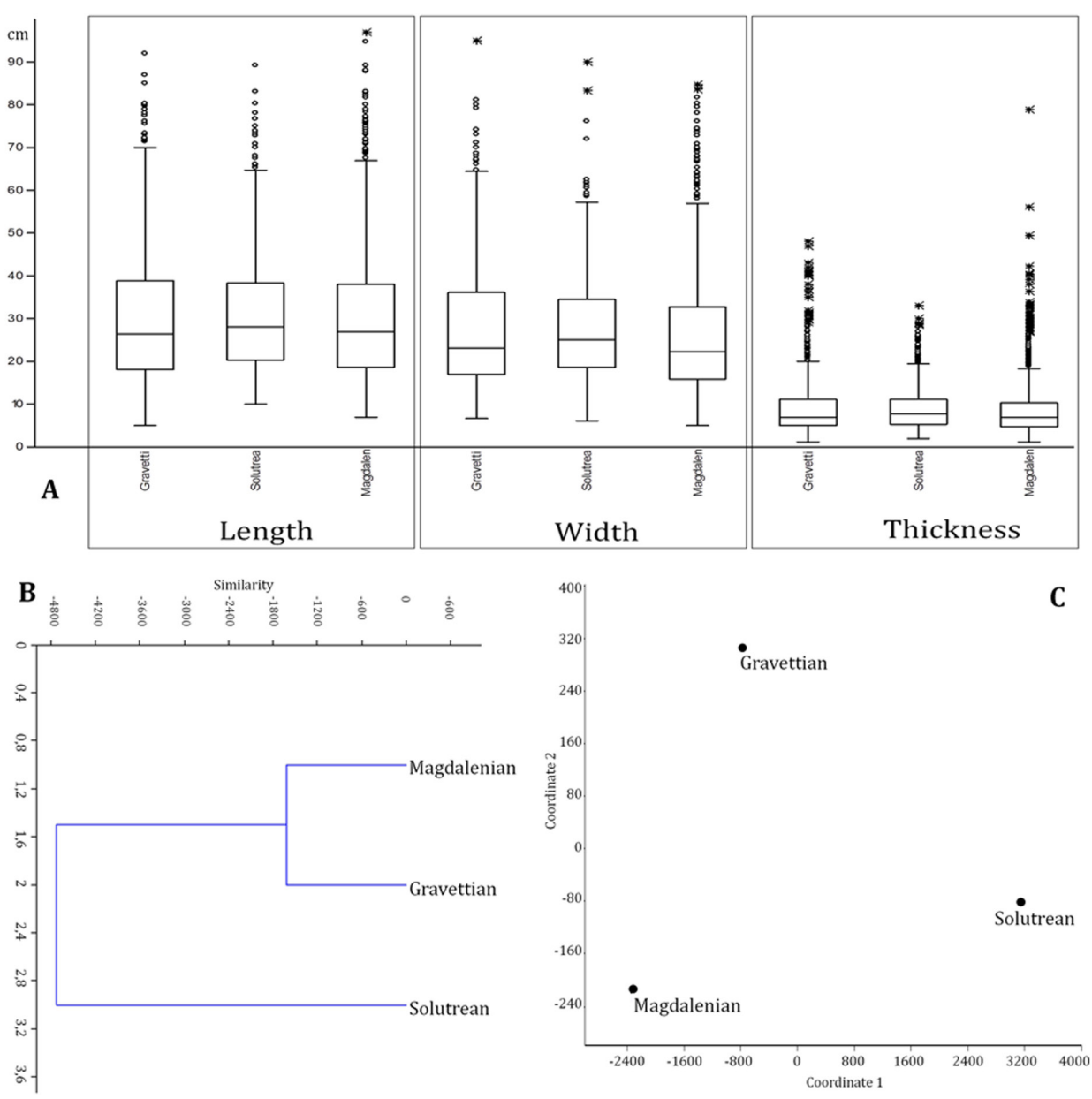

Fig. 10. Quartzite flakes production during the Upper Paleolithic of Southwest Iberian Peninsula: A. Box-plot of flake dimensions (in mm); B. Cluster analysis based on the Euclidean distance between groups using measurements; C. Principal Coordinates analysis using measurements.

mon. While the denticulates decreased substantially, sidescrapers increased (17.4\%), again with simple forms being the most frequent. Considering all the Solutrean quartzite assemblages from SW Iberia, it is striking that only two Laurel Leaf points were identified: one from the Middle Solutrean site of Vale Almoinha and the other from the Upper Solutrean layer from the Slope area in Vale Boi. Finally, during the Magdalenian, tools are more frequent than in any other period. Despite this, assemblages are still dominated by notches, retouched flakes or fragments and denticulates, with sidescrappers decreasing slightly $(15.3 \%)$. Again, the cluster analysis on the tool assemblages group Magdalenian with Gravettian, indicating that these two have more in common than Solutrean (Fig. 12A). This result is corroborated by the Principal Coordinates analysis (Fig. 12B).

\section{DISCUSSION}

Traditionally, European Upper Paleolithic research paid more attention to those lithic assemblages made on flint than other in other raw materials. This is because this flint is the raw material most used for the diagnostic retouched tools and elongate assemblages. This over-attention to flint artifacts denied importance to other raw materials such as quartz and quartzite, resulting in a truncated interpretation. Consequent- 

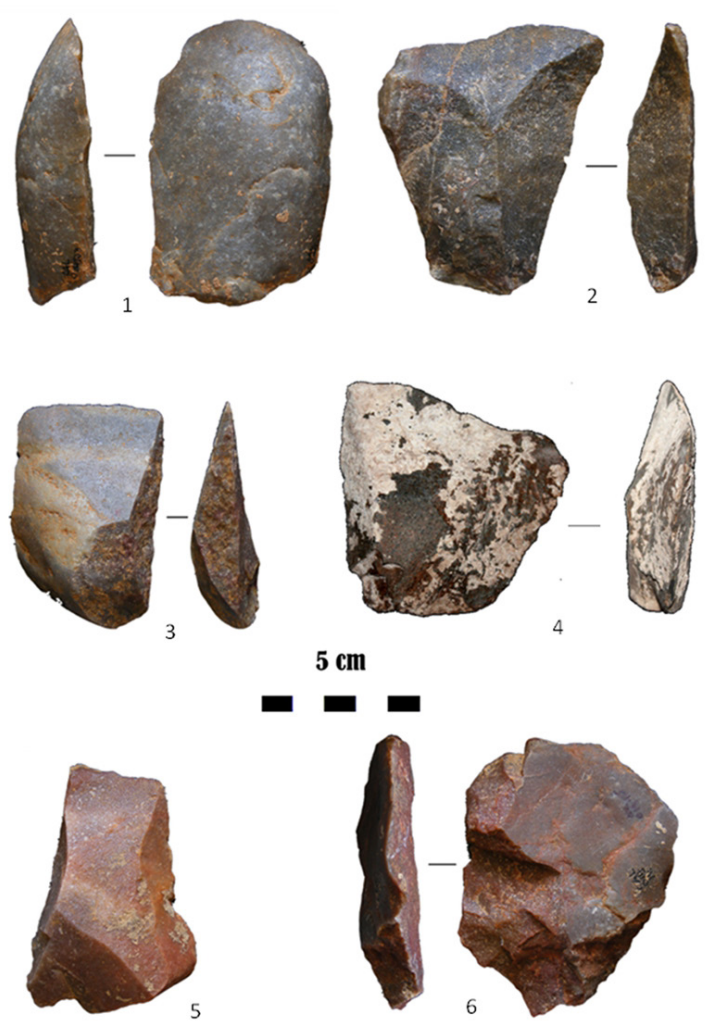

Fig. 11. Typical Solutrean quartzite flakes with sharp edges (5 and 6, burned): 1, 2, 3, 5 and 6 Caldeirão Cave (Tomar), 4 from Vale Boi (Vila do Bispo).

ly, the role of quartzite exploitation was always down-played in the Upper Paleolithic and has never been the focus of in-depth research.

The constant presence of quartzite and greywacke in the SW Iberian Upper Paleolithic assemblages, independent of the distance to flint outcrops, clearly indicates that it had a more relevant role than previously thought. Considering the archaeological evidence, it seems clear that quartz and flint assemblages are more similar to each other than with those of quartzite. Both present, systematically, three different strategies of bladelets production. One based on the reduction of prismatic cores to produce flat profile blanks, other based on the reduction of thick-nose endscrappers for twisted blanks and, finally, a third based on the reduction of burins for the obtainment of thick bladelets, usually known as

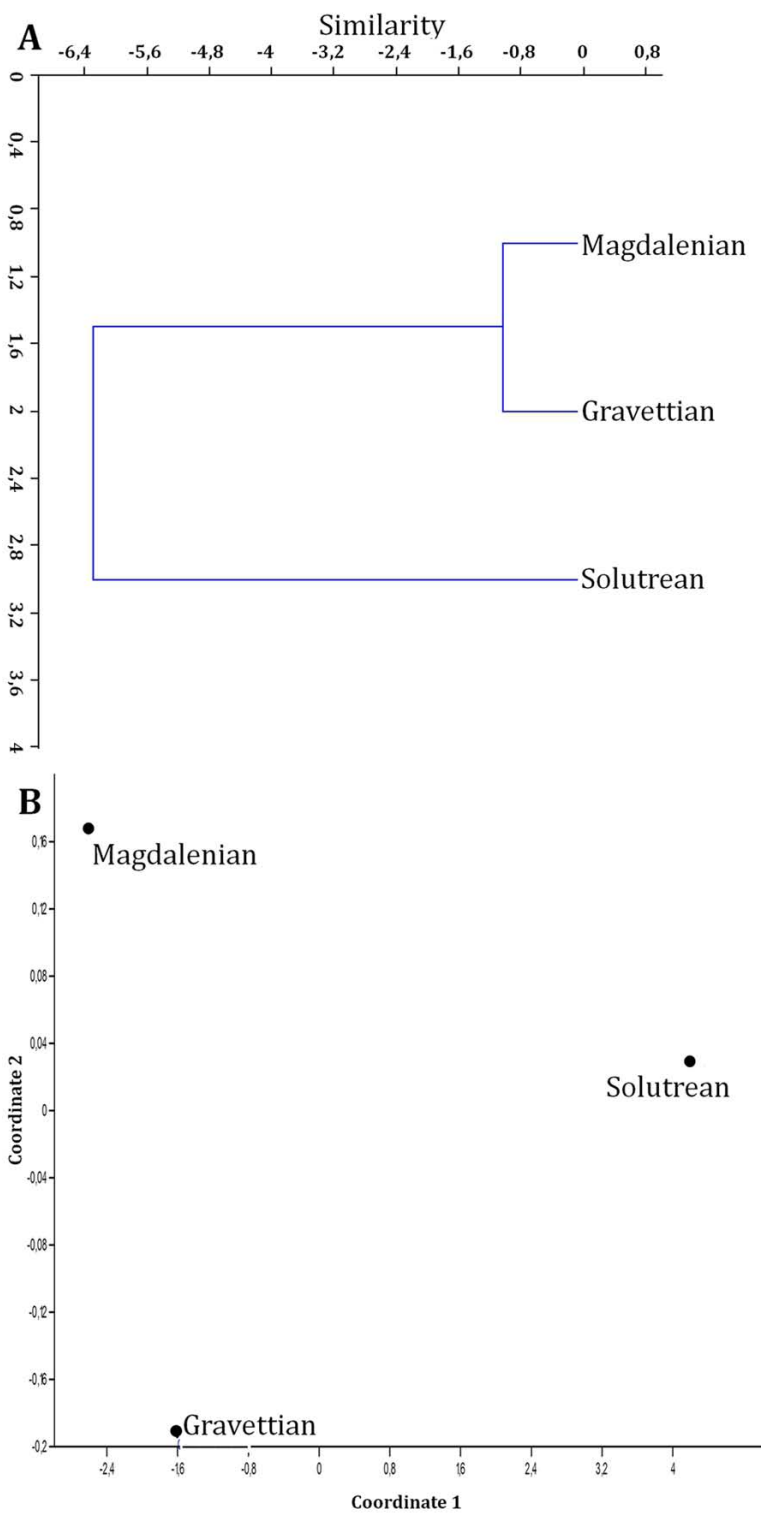

Fig. 12. Quartzite tools during the Upper Paleolithic of Southwest Iberian Peninsula: A. Cluster analysis based on the Euclidean distance between groups using tool assemblages; B. Principal Coordinates analysis using tool assemblages.

burin spalls (Zilhão 1997; Bicho 2000; Marreiros 2009; Mendonça 2009; Cascalheira 2010). These blanks were often retouched into similar spectra of retouched tools, most of them widely interpreted as hafted elements used in many activities but many of them clearly as hunting gear (Zilhão 1997; Bicho 2000), as shown by some use-wear 
analysis (Bicho et al. 2009; Igreja 2009; Marreiros 2009). A very important flake component is also present in both raw materials. The major difference between these assemblages is the relative presence of blades, retouched tools on blades and shaped tools, such as Laurel Leaves. The resemblance between flint and quartz bladelet and flake assemblages is most probably related with the similarity in the physical properties of these two raw materials, since both present a large capacity to produce sharp edges. Furthermore, since the most readily available quartz in SW Iberia tends to split in smaller chunks, the presence of quartz blades is rare. However, when this raw material presents good knapping quality, which is usually associated with macro-crystalline quartz, the resemblance increases considerably because quartz blades can occur (Zilhão 1997; Bicho 2000; Almeida et al. 2004; Marreiros 2009; Mendonça 2009; Cascalheira 2010). On the contrary, as it is shown in this paper, quartzite assemblages present a completely diverse strategy of exploitation, with different pattern of blank and retouched tool production aimed for the production of flakes. Usually, chopper-like cores and flakes compose these assemblages, both with considerable amounts of cortex. The retouch, despite rare, tend to be simple, forming notches, denticulates, retouched flakes and sidescrapers.

The reasons beneath such difference were traditionally related with the physical properties of this raw material. According to some authors (Bicho 1996, 2000; Zilhão 1996, 1997), the coarse trait of quartzite disable it from the knapping ability to produce elongate, thin, sharpedged blanks and highly shaped tools. A detailed observation of the bibliography on the Paleolithic lithic assemblages from SW Iberia gives us strong clues on why this assumption can no longer be considered as valid.

The good knapping features of the SW Iberia quartzite are shown by the existence of recurrent thin Micoquian handaxes (Raposo 1996; CunhaRibeiro 2000; Marks et al. 2002) and Mousterian Levallois flakes and points (Marks et al. 1998; Raposo and Cardoso 1998; Cardoso et al. 2002). The few Solutrean Laurel Leafs (Zilhão 1997; Gibaja and Bicho 2006) and Upper Paleolithic to Epipaleolithic blade and bladelet technology (Zilhão 1997; Quelhas 1999; Bicho 2000; Marreiros 2009; Cascalheira 2010) indicates that this raw material kept its knapping features after the Middle to Upper Paleolithic transition.

Consequently, the almost absence of quartzite retouched tools (except in some random occasions, probably related with unexpected situations) has to be interpreted as an anthropologic result and a cultural option. In other words, tools were not produced in quartzite either because SW Iberian anatomically modern humans did not want to, or because they lacked the ability to work it properly. If the second hypothesis is true, then what could have been the reason for these populations to use it through all the Upper Paleolithic and continued to use it until knapped stone tools were definitely discarded from the tool-kits?

An important indication of the role of quartzite in SW Iberia might come from Central Portuguese region of Estremadura and the Southern coast region of Algarve. Estremadura is highly abundant in very good quality quartz, quartzite and flint, which appear in both primary and secondary deposits. Despite the flint quality and abundance, most Upper Paleolithic sites present a considerable amount of quartzite even when the occupations are located less than $5 \mathrm{Km}$ from flint outcrops. The presence of quartzite is clearly evident especially in those interpreted as residential sites, such as Cabeço de Porto Marinho (Bicho 1996, 2000; Zilhão 1996, 1997). Similarly, Algarve is also highly abundant in quartz, greywacke and flint, however all of lower quality than in Estremadura. Quartzite occurs in very small, rounded and friable pebbles, but there is another coarse raw material that is very abundant in cobbles, pebbles and slabs: greywacke. This raw material appears in all sites that are not flint outcrops and it is particularly abundant through all sequence and in all loci of Vale Boi, a residential site with $\sim 10,000 \mathrm{~m}^{2}$. Reduction patterns of this raw material aimed for the production of flakes in similar quantities and characteristics as those in quartzite on Estremadura (Marreiros 2009; Mendonça 2009; Bicho et al. 2010; Cascalheira 2010; Pereira 2010).

The almost absence of quartzite from the hunting gear of SW Iberian Upper Paleolithic seems to indicate that the absence of use of this raw material in the hunting gear was likely a cultural option. In fact, many hunting tools were pre-determined and sometimes just slightly retouched as is the case of the Vale Comprido and Casal Filipe points. At the same time, quartzite

Trab. Prehist., 69, N. ${ }^{\circ}$ 2, julio-diciembre 2012, pp. 232-256, ISSN: 0082-5638

doi: $10.3989 /$ tp.2012.12090 
retouched tools fit in what is widely known as domestic tools (notches, denticulates, marginal trimmed flakes, sidescrappers, anvils, etc.). This is congruent with the few available use-wear analysis preformed in some Portuguese sites, where quartzite is associated with the procession of vegetable, wood, hide, antler and bone, but not hunting (Carvalho 2007; Bicho et al. 2009; Igreja 2009; Pereira et al. 2011). Quartzite is also highly abundant in sites where intensive carcass and hide processing occurred, such as the Gravettian EE15 occupation layer of Lagar Velho Rockshelter (Almeida et al. 2009) or the Epipaleolithic multi-component site of Barca do Xerêz (Araújo and Almeida 2007).

Together, the available data seem to point to the possibility of coarse-grained raw materials such as quartzite and greywacke to have had a specific role in the everyday SW Iberian Upper Paleolithic activities different from that of just save or replace flint, as it was firstly suggested (Zilhão 1997). This role seems to have been that related with domestic activities and not hunting as argued before by Bicho (1992). Hunting tools are always in flint and quartz. This seems to indicate that was probably quartz and not quartzite that was used to replace flint as previously suggested (Bicho 2000); moreover because flint and quartz allow the production of edges that are more similarly sharp than those in quartzite. This suggests that occupation in fluvial terraces similar to that of Barca do Xerêz (Araújo and Almeida 2007), rich in quartzite chopper and flakes, lacking or poor in flint and quartz hunting gear, might also represent butchering and/or hide process sites. Such dichotomy between the use of flint and quartz in the hunting gear vs. quartzite and greywacke in processing activities seem, at this point, to be explained only with the cultural background of the modern human populations living in the Western Iberian sector between the South margin of the Douro River and Algarve. This situation is clearly different from that seen in most of Iberia, especially Eastern and Mediterranean, where flint dominates over $90 \%$ of the collections. Flint is less present is other Iberian regions, but that seems to be related with its low availability. North of Douro valley and most of Galicia, quartz and quartzite are abundant and flint is rare. This geologic fact couples with the topographic features of this territory (highly mountainous) which event today limits the circu- lation of people through the landscape. Archaeological lithic assemblages in this territory are almost all in quartz and quartzite. Presently, it is assumed that, during the Paleolithic, distance and orography were probably major facts that, together, jeopardized the long distance acquisition of flint (Straus 1980; Utrilla 1981; Arrizabalaga 1999; Llana and Villar 1996).

Another example came from Asturias, a region where flint, quartz and quartzite are abundant but where the Upper Paleolithic assemblages are considerably different from those of SW Iberia. Here, quartzite is present in curated tools (namely projectiles), such as the Solutrean Concave Base points along with domestic tools such as notches, denticulates or retouched flakes (Straus 1980, 1983, 1986; 1996; Cabrera Valdés 1984; Straus and Clark 1986; Rasilla Vives 1989; Bernaldo de Quiros and Cabrera Valdes 1996).

The diachronic variability on the reduction sequences in quartzite identified during the Upper Paleolithic of SW Iberia deserves some discussion. First, from a geological point of view, it seems that there were any changes in the quality, morphology or availability of quartzite during this time span that could explain the existence of such variations. Secondly, the setting of each occupation (open air, rockshelter or cave) might have had a direct relation with the functionality of the occupations and, therefore, with the frequency of each raw material or the spectrum of tools, as it was shown by the butchering sites discussed above. However, it does not seem to have had any influence on the technologic patterns on which the quartzite assemblages were produced. These two facts putted together suggest that the diachronic changes recognized in the quartzite Upper Paleolithic assemblages from SW Iberia have to be related with either the adaptation to raw material constrains or with cultural grounds. The fact that Estremadura is highly rich in good quality flint, quartz and quartzite, seems to set aside the first hypothesis.

Techno-typological approaches to lithic assemblages dated from the Upper Paleolithic of Western Iberian have been performed since early 1990s. They had arrant results on the recognition of idiosyncrasies that enable the distinction of Gravettian, Solutrean and Magdalenian assemblages in both quartz and flint. Therefore, it should not be a surprise that such idiosyncrasies would also be recognized in quartzite. The fact 
that, statistically, both technological traits and retouched tools variability in this raw material always clustered Gravettian with Magdalenian indicates that these resemble each other more than with those from Solutrean. A considerable similarity between Gravettian and Magdalenian assemblages was already recognized for the lithic assemblages in quartz and flint, in such a way that they were only possible to distinguish after absolute dates (3). At the same time, a recent approach to Solutrean assemblages (Cascalheira 2010) indicates that during this period there appears to have been a higher standardization on the lithic production, especially in quartz and flint. The higher standardization recognized in the quartzite flakes is congruent with that. Solutrean quartzite flake assemblages also stand out from the Gravettian and Magdalenian ones for being slightly bigger. Together, the analysis of quartzite flake attributes clearly indicates a consistence shift from Gravettian to Solutrean and from Solutrean to Magdalenian. The fact that quartzite exploitation variability during the three major periods of the SW Iberian Paleolithic closely followed the shifts recognized in the other most used raw materials (flint and quartz) indicates that quartzite use was not occasional but an organic part of the everyday lifestyle.

\section{CONCLUSIONS}

With this study, we were able to accomplish the three objectives proposed. We showed that quartzite had a major importance for the anatomically modern human groups from SW Iberia. This statement is supported by several data. First, quartzite artifacts rarely fit into the hunting-gear and present characteristics that seem to putt such assemblages closer to the domestic activities. This idea seems to be reinforced by the few usewear studies that associate its use to processing activities. Furthermore, the lack of preparation of both cores and blanks, indicate that tools and blanks were probably aimed to be used in the hand and not hafted or as projectiles. Consequently, its use seems to have been complementary to the flint and quartz assemblages; these two with a clear hunting goal despite some domestic ones

(3) Silva, C. 2003: O Olival Fechado no contexto peninsular. Unpublished Master thesis on Prehistory and Archaeology, University of Lisbon. as well, while quartzite was aimed only towards the domestic tasks. This means that it was not used only in an opportunistic fashion or to save flint, but it was part of the daily tool-kit.

Our results also seem to show that the production of these core-and-flake assemblages followed different strategies through time, with consequent different results. Those differences are such that it is now possible to fit each assemblage in the three specific time phases that correspond to Gravettian, Solutrean and Magdalenian without the need of the traditional type-fossils or other dating methods. This is particularly relevant for large areas of Western Europe, from where many sites with rich quartzite (or other coarse raw material) component, lacking traditional type-fossils and that were never dated are known.

Distinction between the Upper Paleolithic sub-periods can be made on pebble/cobble selection, core type, reduction sequences and tool assemblage. Upper Paleolithic quartzite assemblages from SW Iberia can be organized as follow:

Gravettian: pebbles/cobbles chosen for reduction tend to be big, angular, with the later splintered prior the intensive reduction, within the extensive concept. Stepped strategy dominates the assemblages. Extensive cores are only present in this period. This is the period when elongate blanks are rarer, despite that bladelets are more common than blades and where quartzite flakes are smallest;

Solutrean: cores are always in spherical or sub-prismoidal pebbles, never in cobbles. Prismatic and Stepped strategies are dominant. Predeterminate flakes, with $\sim 8 \mathrm{~cm}$ in length, thin, on-axis, with lateral sharp edges and feathered ending can be considered as chronological marker for Solutrean quartzite exploitation. This is the period when elongate blanks are most common, with a relative higher abundance of blades than bladelets. This is the period with more relative frequency of quartzite blades during the all Upper Paleolithic and where quartzite flakes are biggest;

Magdalenian: cobbles/pebbles tend to be large and angular, with a wide platform. Prismatic and Stepped strategies dominate the assemblages. Typical prismatic cores for bladelets occur. Massive tools and flakes with intense edge damage are common. Polyhedric cores are only present in this period. Elongate blanks are rare, but bladelets are more common than blades. Also, this is 
the period with more relative frequency of bladelets and where some quartzite flakes are clearly bigger that average, usually presenting intensive edge damage.

The use of different raw materials in regions with mixed geological features is a transversal phenomenon to geography and chronology. It represents both a simple and effective way to exploit available resources. The results of our study show that the combination of detailed attribute analysis, refitting and use-wear allow to overpass the absence of traditional type-fossils, usually associated with the hunting-gear. Therefore, it should represent a methodological approach to all territories and assemblages, especially to those where such artifacts are often absent. In addition, the possibility of differentiated use of raw materials should be kept open and further tested in order to research to get a deeper understanding of past population behavior. That should include both not archaeological and actualistic studies, once present hunter-gatherer communities are still used as proxy to Pre-historic periods. The possibility of differentiated use of raw materials towards specific goals might, eventually in the future, allow the recognition of some paleoethnographic sub-regions.

\section{ACKNOWLEDGMENTS}

The results here presented resulted in part from the doctoral dissertation of the first author funded by the Portuguese Science Foundation between 2007 and 2010 with the grant reference SFRH/BD/28296/2006. We would like to thank Henrique Matias and Rui Carvalho, without whom the refitting and new interpretation of Terra do Manuel would have been impossible, as well as to Trenton Holliday, Vera Aldeias and the revisers for the comments on the manuscript.

\section{BIBLIOGRAPHY}

Almeida, F. 2000: The Terminal Gravettian of Portuguese Estremadura. Technological variability of the lithic industries. Doctoral dissertation. Southern Methodist University. http://www.academia.edu/ 229888/The Terminal Gravettian of Portuguese Estremadura_Technōogical_variabīitity_of the lithic industries (accessed 22-I-2013).
Almeida, F.; Angelucci, D. E.; Gameiro, C.; Correia, J.; Pereira, T. 2004: "Novos dados para o Paleolítico Superior final da Estremadura portuguesa: resultados preliminares dos trabalhos arqueológicos de 1997-2003 no Lapa dos Coelhos (Casais Martanes, Torres Novas)". Promontoria 2 (2): 157-192.

Almeida, F.; Gameiro, C. and Zilhão, J. 2002: "The Artifact Assemblages". In J. Zilhão and E. Trinkaus (eds.): Portrait of the artist as a child. The Gravettian human skeleton from the Abrigo do Lagar Velho and its archeological context. Trabalhos de Arqueologia 22, Instituto Português de Arqueologia. Lisboa: 202-219.

Almeida, F.; Gameiro, C.; Pereira, T. and Quelhas, A. 2008: "O Paleolítico Superior". In F. Almeida (ed.): Testemunhos do Paleolítico no Regolfo do Alqueva: Resultados do Bloco B1 do Plano de minimização de Impactes sobre o Património Arqueológico. EDIA. Beja: 73-213.

Almeida, F.; Moreno-García, M. and Angelucci, D. 2009: "From under the bulldozer's claws: the EE15 Late Gravettian occupation surface of the Lagar Velho rock-shelter". World Archaeology 41 (2):242261.

Araújo, A. and Almeida, F. 2007: "Inland insights into the Macrolithic puzzle: the case of Barca do Xerez de Baixo". In N. Bicho (ed.): Proceedings of the IV Congresso de Arqueologia Peninsular. From the Mediterranean basin to the Portuguese Atlantic shore: papers in honor of Anthony Marks (Faro 2004): 185-208. Faro.

Arrizabalaga, A. 1999: "El aprovisionamiento en materias primas líticas durante la génesis del Leptolítico: el Cantábrico oriental". Rubricatum 2: 97-104.

Aubry, T. 2009: 200 séculos da história do Vale do Côa: incursões na vida quotidiana dos caçadores - artistas do Paleolítico. Trabalhos de Arqueologia 52, IGESPAR, IP. Lisboa.

Benito del Rey, L. and Benito Álvarez, J. M. 1998: Métodos y materias instrumentales en Prehistoria y Arqueología (la edad de la piedra tallada más antigua), II Tecnología y tipología. Librería Cervantes, Salamanca.

Bernardo de Quiros, F. and Cabrera Valdes, V. 1996: "Raw material in the Palaeolithic of Cueva del Castillo and the Cantabrian Region". In N. Malony, M. Raposo and M. Santonja (eds.): Non-flint stone tools and the Palaeolithic occupation of the Iberian Peninsula. British Archaeological Reports, International Series 649, Archaeopress. Oxford: 21-32.

Bicho, N. 1992: "Technological change in the final Upper Paleolithic of Rio Maior, Portuguese Estremadura". Doctoral dissertation. Southern Methodist University.

Bicho, N. 1996: "The role of quartz and quartzite in the Magdalenian of Cabeço de Porto Marinho, Rio Maior, Portugal”. In N. Maloney, L. Raposo and M. 
Santonja (eds.): Non-flint stone tools and the Palaeolithic occupation of the Iberian Peninsula, British Arcaheological Reports International Series 649, Archaeopress, Oxford: 175-181.

Bicho, N. 1997: "A ocupaçao epipaleolítica do Abrigo Grande das Bocas, Rio Maior”. O Arqueólogo Português 4 (13-15): 53-85.

Bicho, N. 2000: Technological Change in the Final Upper Paleolithic of Rio Maior. Portuguese Estremadura. CEIPHAR. Tomar.

Bicho, N. 2001: "Sistemas de povoamento, mobilidade e aproveitamento dos recursos naturais no território português durante a transição PlistocénicoHolocénico". Revista Lusíada 1: 31-58.

Bicho, N. 2004: "As comunidades humanas de caçadores-recolectores do Algarve Ocidental: Perspectiva ecológica". In A. Tavares, M. Tavares and J. Cardoso (eds.): Evolução geohistórica do litoral português e fenómenos correlativos. Universidade Aberta. Lisboa: 359-396.

Bicho, N.; Hawks, J.; Hockett, B.; Marlova, A. and Belcher, W. 2003: "Paleocologia e ocupaçao humana da Lapa do Picareiro: resultados preliminares". Revista Portuguesa de Arqueologia 6 (2): 49-81.

Bicho, N.; Hawks, J.; Gibaja, J. and Hockett, B. 2009: "Lapa do Picareiro, un asentamiento de caza magdaleniense en la Estremadura potuguesa". Complutum 20 (1): 71-82.

Bicho, N.; Manne, T.; Casacalheria, J.; Marreiros, J.; Mendonça, C.; Évora, M.; Gibaja, J. and Pereira, T. 2010: "O Paleolítico Superior do Sudoeste da Península Ibérica: o caso do Algarve". In X. Mangado (ed.): El Paleolítico Superior peninsular. Novedades del siglo XXI. Homenaje al Profesor Javier Fortea. Monografies 8, Universitat de Barcelona, SERP. Barcelona: 219-238.

Bordes, F. 1961: "La typologie du paléolithique ancien et moyen". CNRS, Paris.

Breuil, H. and Zbyszewski, G. 1942: "Contribution a l'étude des industries paléolithiques du Portugal et de leurs rapports avec la géologie du Quaternaire. Les principaux gisements des deux rives de l'ancien estuaire du Tage". Comunicações dos Serviços Geológicos de Portugal 23: 1-369.

Breuil, H. and Zbyszewski, G. 1945: "Contribution a l'étude des industries paléolithiques du Portugal et de leurs rapports avec la géologie du Quaternaire. Les principaux gisements des plages quaternaires du littoral d'Estremadura et des terraces fluviales de la basse vallé du Tage". Comunicações dos Serviços Geológicos de Portugal 26: 1-678.

Brézillon, M. 1983: La Dénomination des objets de pierre taillée. Matériaux pour un vocabulaire des préhistoriens de langue française. IV Supplément a Gallia Prehistoire. CNRS. Paris.
Cabrera Valdes, V. 1984: El yacimiento de la cueva del Castillo (Puente Viesgo, Santander). Bibliotheca Praehistorica Hispana XXII, CSIC. Madrid.

Cardoso, J. L.; Raposo, L. and Ferreira, O. V. 2002: A Gruta Nova da Columbeira: Bombarral. Câmara Municipal do Bombarral. Bombarral.

Carvalho, A. F. 2007: A neolitização do Portugal meridional os exemplos do Maciço Calcário Estremenho e do Algarve Ocidental. Promontória Monográfica 12, University of Algarve. Faro.

Cascalheira, J. 2010: Tecnologia lítica solutrense do Abrigo de Vale Boi (Vila do Bispo). Cadernos da UNIARQ 5, Universidade de Lisboa. Lisboa.

Costa, J. 1998: Estudo e classificação das rochas por exame macroscópico. Serviço de Educação, Fundação Calouste Gulbenkian. Lisboa.

Cunha-Ribeiro, J. P. 2000: “A indústria litica Do Casal do Azemel no contexto da evoluçao do Paleolítico inferior na Ibéria Ocidental". In V. Oliveira Jorge (ed.): Actas do $3 .{ }^{\circ}$ Congreso de Arqueologia Peninsular II, Paleolítico da Península Ibérica: 137-168 Porto.

Farndon, J. 2006: The Complete Guide to Rocks and Minerals. Hermes House. London.

Gameiro, C. and Almeida, F. 2001: "A indústria de pedra lascada da camada TP06 do Testemunho Pendurado do Abrigo do Lagar Velho (Lapedo, Caranguejeira, Leiria), no contexto da passagem do Gravetense para o Solutrense na Estremadura Portuguesa". In A. Figueiredo, G. Oliveira and C. Carvalho (eds.): Actas das I Jornadas de património e Arqueologia do litoral centro: 7-27. Leiria.

Gibaja, J. and Bicho, N. 2006: "La función de los instrumentos líticos en el asentamiento de Vale Boi (Algarve, Portugal). Estudio del utillaje Gravetiense y Solutrense. Saguntum 38: 9-21.

Heleno, M. 1956: "Um quarto de século de investigação arqueológica". O Archeologo Português, 2. ${ }^{\circ}$ Série 3: 221-237.

Igreja, M. 2009: "Estudo traceológico das industrias líticas de Olga Grande 4 e Cardina I: Funçao, modo de funcionamento dos artefactos e outras inferências paleocomportamentais". In T. Aubry (ed.): 200 séculos da historia do Vale do Côa: incursoes na vida quotidiana dos caçadores-artistas do Paleolítico. Trabalhos de Arqueologia 52, IGESPAR, IP. Lisboa: 235-246.

Inizan, M. L.; Reduron-Ballinger, M.; Roche, H. and Tixier, J. 1999: Technology and terminology of knapped stone. Préhistoire de la pierre taillée 5, Cercle de Recherches et d'Études préhistoriques. Nanterre.

Llana, C. and Villar, R. 1996: "Quartz and quartzite industries in the Upper Palaeolithic of Galicia and Asturias: the relationship between morphostructure, technical characteristics and typology". In N. Maloney, L. Raposo and M. Santoja (eds.): Non-flint stone tools and the Palaeolithic occupation of the 
Iberian Peninsula. British Archaeological Reports, International Series 649, Archaeopress. Oxford: 4347.

Marks, A.; Bicho, N.; Zilhão, J. and Ferring, C. 1994: "Upper Pleistocene Prehistory in Portuguese Estremadura. Results of Preliminary Research". Journal of Field Archaeology 21: 53-68.

Marks, A; Brugal, J.; Chabai, V.; Monigal, K; Goldberg, P.; Hockett, B.; Peman, E.; Elorza, M. and Mallol, C. 2002: "Le gisement Pléistocène moyen de Galeria Pesada (Estrémadure, Portugal): premiers résultats". Paleo 14: 77-100.

Marks, A.; Monigal, K. And Zilhão, J. 1998: "The lithic assemblages of the Late Mousterian at Gruta da Oliveira, Almonda, Portugal". In J. Zilhão, T. Aubry and A. Carvalho (eds.): Les premiers homes moderns de al Péninsule Iberique. Instituto Portugês De Arqueologia. Lisboa: 145-154.

Marreiros, J. 2009: As primeiras comunidades do Homem moderno no Algarve Ocidental: Caracterização paleotecnológica e paleoetnográfica das comunidades gravetenses e proto-solutrense de Vale Boi (Algarve, Portugal). Master thesis. University of Algarve, Faro. http://hdl.handle. net10400.1/314 (accessed 5-VI-2012).

Meireles, J. 1992: As indústrias líticas do litoral minhoto. Contexto cronoestratigráfico e paleoambiental, Cadernos de Arqueologia, Monografias 7, Universidade do Minho. Braga.

Mendonça, C. 2009: A tecnologia lítica no tardiglaciar do Algarve. Masters thesis. University of Algarve. Faro. htpp://hdl.handle.net/10400.1/819 (accessed 5-VI-2012).

Pereira, T. 2010: A exploração do quartzito na Faixa Atlântica Peninsular durante o final do Plistocénico. Doctoral dissertation. University of Algarve, Faro. http://ualg.academia.edu/TelmoPereira/ Books/1287048/2010_A_exploracao_do_quartzito na_Faixa_At Tantica_Peninsular_no_Final_do Plistocenico (accessed $\overline{4}-\mathrm{V}-2012)$.

Pereira, T.; Gibaja, J.; Almeida, F.; Holliday, T. y Bicho, N. 2011: How they did it: Gravettian quartzite flakes from Western Iberia. In A. Pastoors and M. Peresani (eds.): In Flakes not Blades. Dusseldorf: Stiftung Neanderthal Museum.

Preston, Neil J. 1988: "The Triassic Bunter Pebble Beds as a Source for Non-Flint Artefacts in the Upper Thames Basin". In R. J. MacRae and N. Moloney (eds.): Non-Flint Stone Tools and the Palaeolithic Occupation of Britain, British Archaeological Reports, International Series 189, Archaeopress. Oxford: 199-216.

Quelhas, A. 1999: “A estaçao do Magdalenense Final da Bairrada (Torres Novas). Caracterizaçao tipológica, tecnológica e cultural". Revista Portuguesa de Arqueologia 2 (2): 15-29.
Raposo, L. 1986: "Mustierense, Mustiero-Languedocense ou Languedocense". Arqueologia 14: 13-21.

Raposo, L. 1996: "Quartzite bifaces and cleavers in the final Acheulean assemblage of Milharós (Alpiarça, Portugal)". In N. Maloney, L. Raposo and M. Santonja (eds.): Non-flint stone tools and the Palaeolithic occupation of the Iberian Peninsula. British Archaeological Reports, International Series 649, Archaeopress. Oxford: 151-165.

Raposo, L. and Cardoso, J. 1998: O Paleolítico Médio da Conçeiçcao (Al cochete). Lusoponte/CEMA. Lisboa.

Raposo, L. and Silva, A. 1984: “O Languedocense: ensaio de caracterização morfotécnica e tipológica”. O Arqueólogo Português, Série IV: 87-166.

Rapp, G. and Hill, C. 1998: "Geoarchaeology”. Yale University Press. New Haven.

Rasilla Vives, M. de la 1989: "Sequencia y cronoestratigrafia del Solutrense cantábrico". Trabajos de Prehistoria 46: 35-46.

Ribeiro, A.; Antunes, M. T.; Ferreira, M. P.; Rocha, R. B.; Soares, A. F.; Zbyszewski, G.; Moitinho De Almeida, F.; Carvalho, D. and Monteiro, J. H. 1979: "Introduction à la géologie générale du Portugal". Serviços Geológicos de Portugal, Lisboa.

Ribeiro, C. 1871: “Descripção de alguns silex e quartzites lascados encontrados nas camadas dos terrenos terciario e quaternário das bacias do Tejo e do Sado". Memória apresentada à Academia Real das Sciencias de Lisboa. Lisboa.

Sonneville-Bordes, D. and Perrot, J. 1954: "Lexique typologique du Paléolithique supérieur". Bulletin de la Société Préhistorique Française 51: 327-335.

Sonneville-Bordes, D. and Perrot, J. 1955: "Lexique typologique du Paléolithique supérieur". Bulletin de la Société Préhistorique Française 52: 76-79.

Sonneville-Bordes, D. and Perrot, J. 1956: "Lexique typologique du Paléolithique supérieur". Bulletin de la Société Préhistorique Française 53: 408-412, 547-559.

Straus, L. 1980: "The role of raw materials in lithic assemblage variability". Lithic Technology 9: 68-72.

Straus, L. 1983: El Solutrense vasco-cantabrico: una nueva perspectiva. Centro de Investigación y $\mathrm{Mu}-$ seo de Altamira Monografías 10, Dirección de Bellas Artes y Archivos. Madrid.

Straus, L. 1986: "A comparison of La Riera assemblages with those from contemporany sites in Cantabrian Spain". In L. G. Strauss y G. A. Clarck (eds.): La Riera Cave. Stone Age Hunter-Garether Adaptations in Northern Spain. Anthropological Research Papers, 36, Arizona State University, Tempe, Arizona: 219-236.

Straus, L. 1996: "The use of quartzite in the Upper Paleolithic of Cantabrian Spain". In N. Maloney, L. Raposo and M. Santonja (eds.): Non-flint stone tools and the Palaeolithic occupation of the Iberian 
Peninsula, British Archaeological Reports, International Series 649, Archaeopress. Oxford: 37-41.

Straus, L. G. and Clark, G. 1986: La Riera Cave. Stone Age Hunter-Gatherer Adaptations in Northern Spain. Anthropological Research Papers 36, Arizona State University: Arizona.

Utrilla, P. 1981: El Magdaleniense Inferior y Medio en la Costa Cantabrica. Centro de Investigación y Museo de Altamira, Monografías 4. Madrid, Santander.

Zbyszewski, G. 1966: "Conhecimentos actuais sobre o Paleolítico português". Comemoração do I Centenário da Associação dos Arqueólogos Portugueses, II, Associação dos Arqueólogos Portugueses, Lisboa: 109-133.
Zilhão, J. 1996: "The use of non-flint raw materials in a late Palaeolithic assemblage from Gruta do Caldeirao (Tomar, Portugal)". In N. Maloney, L. Raposo and M. Santonja (eds.): Non-flint stone tools and the Palaeolithic occupattion of the Iberian Peninsula. British Archaeological Reports, International Series 649, Archaeopress. Oxford: 167-174.

Zilhão, J. 1997: "O Paleolítico Superior da Estremadura portuguesa". Colibri. Lisboa.

Zilhão, J. and Almeida, F. 2002: "The archaeological framework". In J. Zilhão; E. Trinkaus (eds.): Portrait of the artist as a child. The Gravettian human skeleton from the Abrigo do Lagar Velho and its archaeological context. Trabalhos de Arqueologia 22, Instituto Português de Arqueologia. Lisboa: 29-57. 